\title{
DESLOCAMENTOS DA POPULAÇÃO INDÍGENA PARA ACESSO AOS SERVIÇOS DE SAÚDE: ELEMENTOS PARA AÇÕES EMERGENCIAIS DE ENFRENTAMENTO À COVID-19
}

\author{
Fernando Damasco* \\ Universidade Federal Fluminense
}

\section{Marta Antunes**}

Museu Nacional - Universidade Federal do Rio de Janeiro

\section{Marta Azevedo***}

Universidade de Campinas

Resumo: Este artigo apresenta elementos geográficos e estatísticos para subsidiar ações emergenciais de enfrentamento à coVID-19 junto aos povos indígenas. Nele estão reunidos dados sobre deslocamento para acesso a serviços de saúde de baixa, média e alta complexidade (IBGE, 2020b) e estimativas de população indígena residente em Terras e Agrupamentos Indígenas construídas pelos autores a partir de dados do Censo Demográfico 2010 e da Base de Informação sobre Indígenas (IBGE, 2012, 2020a). A partir da avaliação dessas informações sobre a presença nos municípios brasileiros de população residente em terras e agrupamentos indígenas e da existência ou não de polos de alta complexidade nesses municípios, recomendações são apresentadas sobre quais os municípios prioritários para reforçar o atendimento emergencial aos povos indígenas para enfrentamento da COVID-19.

Palavras chave: Atenção Emergencial à Saúde dos Povos Indígenas; Polos de atendimento aos povos indígenas; Covid-19.

\section{Indigenous geographical mobility to access healthcare services: elements for emergency healthcare actions on the fight against COVID-19}

Abstract:Geographical and statistical inputs for decision making regarding emergency healthcare actions for indigenous peoples on the fight against COVID-19 are presented in this article. A set of information on geographical mobility to access healthcare services of high, medium and lower complexity (IBGE, 2020b) and estimates for indigenous population inhabiting Indigenous Reserves and Settlements built by the authors using 2010 Census data and the Indigenous Information Database (IBGE, 2012, 2020b) was combined in order to provide a starting point for analyses. Based on the evaluation of this combined information about the indigenous presence in Brazilian cities and the existence or absence of high level complexity hubs for healthcare in these cities, recommendations indicate the priority municipalities for reinforcement of their infrastructural of emergency healthcare to indigenous in the context of COVID-19.

Keywords: Emergency Health Care for Indigenous People; Hubs of health care for Indigenous people Covid-19.

\section{Movilidad geográfica de la población indígena para acceso a los servicios de salud: elementos para acciones emergencia de combate a la coVID-19}

Resumen: Este artículo presenta elementos geográficos y estadísticos de apoyo a acciones de emergencia en el combate a la COVID-19 junto a los pueblos indígenas. Reúne y analiza datos sobre desplazamiento de esa población para acceder a servicios de salud de baja, media y alta complejidad (IBGE, 2020b), y estimaciones de los totales de población indígena que reside en las Tierras y Agrupamientos Indígenas constituidas por los autores basados en los datos del Censo Demográfico 2010 y en la Base de Informaciones sobre los Indígenas (IBGE, 2012, 2020a). Con base en la evaluación de esta información sobre la presencia de población residente en tierras e y agrupamientos indígenas en los municipios brasileños, y de la existencia, o no, de centros de salud de alta complejidad en esos municipios, se presentan recomendaciones sobre cuáles son los municipios prioritarios para reforzar la asistencia de emergencia a los pueblos indígenas para el enfrentamiento a la COVID-19.

Palabras clave: Atención de emergencia a la salud de los pueblos indígenas; COVID-19; centros de salud para los pueblos indígenas. 
Introdução

Este artigo tem como objetivo contribuir para os programas de prevenção e atendimento ao novo coronavírus - Covid-19 - entre os povos indígenas a partir da avaliação de informações geográficas e estatísticas sobre a presença nos municípios brasileiros de população residente em terras e agrupamentos indígenas. 0 trabalho aqui apresentado incorpora pesquisa publicada em artigo anterior dos mesmos autores (Azevedo et al., 2020) onde demonstramos um ranking de vulnerabilidade à Covid-19 entre as terras indígenas e os Distritos Especiais de Saúde Indígena DSEls - a partir de um indicador criado com elementos da estrutura demográfica, segurança territorial, saneamento e disponibilidade de serviços de emergência com estrutura de Unidades de Tratamento Intensivo - UTIs.

A ideia é que este material ofereça subsídios para as ações das próprias comunidades e organizações indígenas, do governo e outros aliados para o atendimento à saúde indígena nesse período de pandemia que estamos vivendo no Brasil e em todo o planeta. Apontamos principalmente para o dimensionamento de municípios prioritários para a instalação de unidades emergenciais de atenção à saúde.

Foi elaborada uma estimativa da população residente em terras e agrupamentos indígenas por municípios, inclusive nas áreas urbanas, referente ao ano de 2019, com base nos dados da Base de Informações sobre os Indígenas, recentemente divulgada pelo IBGE, e nos dados do Censo Demográfico 2010.

Esses dados foram associados ainda aos resultados da Pesquisa Região de Influência das Cidades - Regic 2018, também recentemente divulgada pelo IBGE, sobre os deslocamentos populacionais para acesso a serviços de saúde de baixa, média e alta complexidade.

0 estudo tem caráter preliminar e sua divulgação atende os objetivos de emergência, considerando-se a velocidade de interiorização da Covid-19 no país. 0 objetivo principal é que o texto seja de amplo acesso e circulação, de modo que contém alguns elementos que podem não atender à totalidade das normas habituais dos artigos científicos.

\section{Conceitos e definições fundamentais}

A população alvo do estudo é aquela residente em terras e agrupamentos indígenas, em áreas urbanas ou rurais, e que aqui designaremos de "população indígena". $\mathrm{Na}$ construção das estimativas populacionais referentes ao ano de 2019 , não foram consideradas nos cálculos as pessoas declaradas ou consideradas indígenas e que residam fora de terras e agrupamentos indígenas.

Como "agrupamento indígena", entende-se aqui:

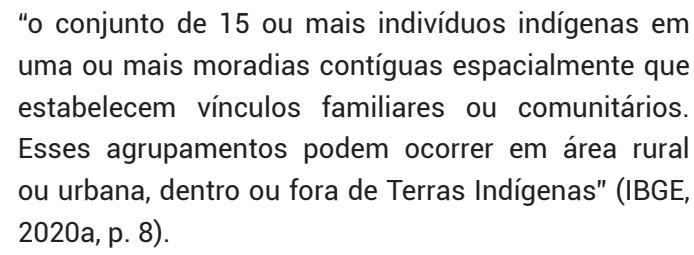

Para fins de cálculo das estimativas populacionais, foram consideradas as terras indígenas oficialmente delimitadas pela FUNAI, independentemente do estágio do processo demarcatório, conforme a metodologia do IBGE (2020a).

São aqui utilizadas também classificações para os municípios estudados que são oriundas do módulo da pesquisa Regic 2018 (IBGE, 2020b), divulgada em caráter emergencial pelo IBGE para enfrentamento à Covid-19. Essa é uma pesquisa que oferece um panorama dos deslocamentos da população entre os municípios ${ }^{1}$ brasileiros para o acesso a serviços de saúde e que categoriza os municípios brasileiros entre as seguintes condições:

- Municípios de destino: aqueles para os quais a população residente se dirige em busca dos serviços de saúde, sem considerar os serviços recebidos no próprio município. São considerados os deslocamentos realizados em busca de atendimento.

- Municípios de origem: aqueles de onde parte a população em busca dos serviços de saúde.

Muitos municípios apresentam as duas condições, tendo em vista que recebem a população de municípios menores, mas, ao mesmo tempo, têm a sua população se deslocando para atendimento em municípios que possuem estrutura para atendimentos de maior complexidade.

Os municípios de destino funcionam como centralidades, que atraem população de outros municípios com base nos serviços de saúde oferecidos e, por isso, aqueles com maior volume de procura são aqui

'A Pesquisa Regic 2018 operacionaliza o conceito de "cidade", para fins da própria pesquisa, a partir das unidades territoriais propostas pelo IBGE em Arranjos Populacionais e Concentrações Urbanas no Brasil (IBGE, 2016). Os centros urbanos pesquisados são definidos a partir da classificação dos municípios em "arranjos populacionais", "concentrações urbanas" e "demais municípios isolados". Entretanto, de modo a simplificar a compreensão deste estudo, os municípios foram referidos genericamente, sabendo-se que, quando mencionamos determinada cidade, estamos nos referindo simultaneamente ao seu enquadramento como arranjo populacional, concentração urbana ou município isolado. Para acesso à classificação de cada município citado neste estudo, consultar IBGE (2016) 
definidos como "polos de atração". A população que tem como destino um mesmo polo ou município de destino será aqui definida como "população dependente", uma vez que se trata da população que depende daquele polo ou município para satisfação de suas necessidades de saúde.

A Regic 2018 classifica ainda os municípios quanto à preponderância do tipo de serviço de saúde prestado pelo município, utilizando a definição de alta, média e baixa complexidade do Ministério da Saúde (2009), que oferece as seguintes concepções:

- Alta complexidade: conjunto de procedimentos que, no contexto do SUS, envolve alta tecnologia e alto custo, objetivando propiciar à população acesso a serviços qualificados, integrando-os aos demais níveis de atenção à saúde;

- Média/baixa complexidade: compõe-se por ações e serviços que visam a atender aos principais problemas de saúde e agravos da população, cuja prática clínica demande disponibilidade de profissionais especializados e o uso de recursos tecnológicos de apoio diagnóstico e terapêutico. Compreendem consultas médicas e odontológicas, exames clínicos, serviços ortopédicos e radiológicos, fisioterapia e pequenas cirurgias, dentre outros atendimentos que não impliquem em internação (IBGE, 2020b).

\section{Metodologia}

A metodologia para análise da distribuição do atendimento à saúde entre a população residente em terras e agrupamentos indígenas contemplou a construção de uma estimativa da população residente nessas áreas para o ano de 2019 e também a correlação destes dados com as informações de deslocamento da população para atendimento e acesso a serviços de saúde de baixa, média e alta complexidade.

A construção das estimativas de população residente em terras e agrupamentos indígenas para o ano de 2019 contemplou inicialmente o cálculo, para cada município, da média de moradores por domicílios em Terras Indígenas, de acordo com dados do Censo Demográfico 2010. Esse valor foi conjugado aos dados de Estimativa de Domicílios Indígenas (EDOC) segundo municípios, provenientes da Base de Informações sobre os Indígenas (IBGE, 2020a).

De acordo com o IBGE, a estimativa de domicílios ocupados (EDOC) é o "quantitativo estimado pelo IBGE de domicílios particulares permanentes ocupados a serem recenseados em determinado recorte territorial" (IBGE, 2020a), sendo alvo de confirmação a cada Censo. Sendo domicílio o local estruturalmente separado e independente que se destina a servir de habitação a uma ou mais pessoas, ou que esteja sendo utilizado como tal e o domicílio particular permanente aquele no qual o relacionamento entre seus ocupantes era ditado por laços de parentesco, de dependência doméstica ou por normas de convivência e que foi construído para servir, exclusivamente, à habitação e, na data de referência, tinha a finalidade de servir de moradia a uma ou mais pessoas e estava ocupado, ou seja, com moradores nele residindo ${ }^{2}$.

Os dados de estimativa de domicílios em áreas indígenas, por municípios, foram multiplicados pela média municipal de moradores por domicílios em terras indígenas do Censo Demográfico 2010, chegando-se assim à estimativa de população residente em terras e agrupamentos indígenas no ano de 2019. Para os municípios em que esta média não estava disponível em 2010, foram utilizadas as médias das Grandes Regiões: 5,7 para a Região Norte, 4,1 para o Nordeste, 4,4 para o Sudeste, 4,5 para o Sul e 5,0 para o Centro-Oeste.

A estimativa da população residente em terras e agrupamentos indígenas pôde, então, ser associada aos resultados da Pesquisa Regic 2018 sobre o acesso da população aos serviços de saúde. Foram utilizados os dados de município de origem e município de destino da compilação "alta, média e baixa complexidade".

Em seguida, foi realizada a hierarquização dos principais polos de destino da população segundo a estimativa de população e representação por meio de cartograma, utilizando-se os arquivos vetoriais da Pesquisa Regic 2018. A partir desses resultados, foi possível avaliar a participação dos municípios de origem no volume de atendimento dos polos de destino da população, o que pode indicar possíveis pontos de instalação emergencial de estruturas de saúde para desafogar os grandes polos e garantir vagas suficientes para o atendimento aos indígenas. 
Tabela 1: Polos de atração (destino) da população residente em Terras e Agrupamentos Indígenas para acesso a serviços de saúde por nível de complexidade, de acordo com a estimativa de população dependente (2019)

\begin{tabular}{|c|c|c|c|c|}
\hline UF & Município & Complexidade & $\begin{array}{l}\text { Estimativa da população } \\
\text { dependente residente em } \\
\text { Tls e agrupamentos }\end{array}$ & Leitos de UTI \\
\hline AM & MANAUS & Alta & 194.885 & 177 \\
\hline RR & BOA VISTA & Alta & 75.450 & 17 \\
\hline PE & RECIFE & Alta & 61.044 & 392 \\
\hline AM & TABATINGA & Baixa média & 59.772 & 0 \\
\hline GO & GOIÂNIA & Alta & 55.485 & 291 \\
\hline PA & SANTARÉM & Alta & 41.100 & 27 \\
\hline MS & DOURADOS & Alta & 38.467 & 46 \\
\hline $\mathrm{PI}$ & TERESINA & Alta & 33.928 & 103 \\
\hline RO & PORTO VELHO & Alta & 30.789 & 118 \\
\hline MS & CAMPO GRANDE & Alta & 30.173 & 106 \\
\hline MT & CUIABÁ & Alta & 28.761 & 93 \\
\hline$A C$ & RIO BRANCO & Alta & 26.836 & 33 \\
\hline BA & SALVADOR & Alta & 15.817 & 425 \\
\hline BA & ITABUNA & Alta & 14.906 & 19 \\
\hline PB & MAMANGUAPE & Baixa média & 14.384 & 10 \\
\hline$A L$ & MACEIÓ & Alta & 13.972 & 88 \\
\hline MT & SORRISO & Alta & 13.001 & 6 \\
\hline PE & SERRA TALHADA & Baixa média & 12.663 & 10 \\
\hline CE & SOBRAL & Alta & 12.476 & 75 \\
\hline MA & IMPERATRIZ & Alta & 11.524 & 52 \\
\hline MG & MONTES CLAROS & Alta & 11.460 & 75 \\
\hline AM & SANTO ANTÔNIO DO IÇÁ & Baixa média & 11.256 & 0 \\
\hline PA & BELÉM & Alta & 10.983 & 144 \\
\hline MT & SINOP & Alta & 9.767 & 6 \\
\hline MT & TANGARÁ DA SERRA & Alta & 9.347 & 0 \\
\hline RS & PASSO FUNDO & Alta & 8.929 & 43 \\
\hline
\end{tabular}




$\begin{array}{lllrr}\text { AM } & \text { TEFÉ } & \text { Baixa média } & 8.847 & 0 \\ \text { AP } & \text { MACAPÁ } & \text { Alta } & 7.265 & 12 \\ \text { SC } & \text { XANXERÊ } & \text { Alta } & 6.851 & 10 \\ \text { PA } & \text { REDENÇÃO } & \text { Alta } & 6.404 & 9\end{array}$

Fonte: Estimativa populacional elaborada pelos autores com base no Censo Demográfico 2010 e na Base de Informações sobre os Indígenas (IBGE, 2012, 2019). Os dados de UTI são provenientes do Cadastro Nacional de Estabelecimentos de Saúde (CNES, 2020).

\section{Resultados}

Os resultados mostram que a população indígena em todo o Brasil se desloca para 143 destinos diferentes para acesso aos serviços de saúde, dos quais 41 não possuem leitos de UTIs disponíveis.

Os dados mostram que Manaus é o principal polo de atração do país, sendo referência para uma população de 194.885 pessoas provenientes de terras e agrupamentos indígenas de diversos municípios do estado do Amazonas, o que representa $19 \%$ de toda a população indígena.

Em seguida estão Boa Vista, capital de Roraima, o Recife, que é polo de atração de grande parte da população indígena do Nordeste, e Tabatinga, que funciona como polo regional, submetida a Manaus.

Importante destacar a centralidade de Dourados em relação à população indígena do Mato Grosso do Sul, absorvendo um contingente de mais de 38 mil pessoas residentes nessas áreas.

$\mathrm{Na}$ tabela 1, são apresentados os dados dos 30 principais polos de atração de população indígena para acesso a serviços de saúde.

$\mathrm{Na}$ figura 1, estão representados os principais polos de atração da população indígena para acesso a serviços de saúde. 
Figura 1: Polos de atração (destino) da população residente em Terras e Agrupamentos Indígenas para acesso a serviços de saúde, por nível de complexidade, de acordo com a estimativa de população dependente (2019)

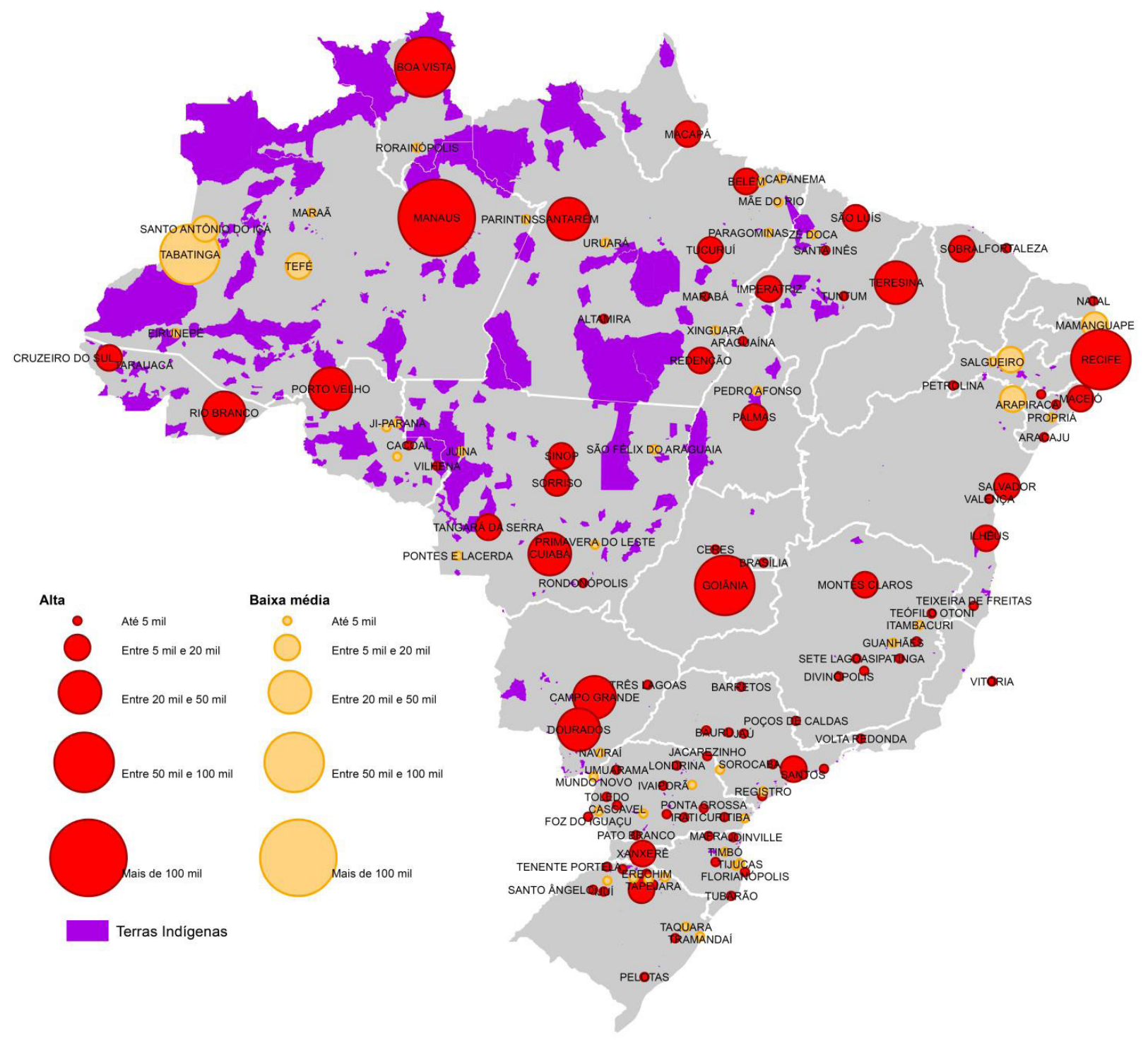

Fontes: Elaborado pelos autores, 2020.

Os dados também foram analisados no sentido de avaliar os municípios com maior contingente de populaçãoresidenteemterraseagrupamentosindígenas. São Gabriel da Cachoeira/AM é o município com mais expressiva população (44.208), seguido do município de Águas Belas/PE, Jacareacanga/PA, Tabatinga/AM e São Paulo de Olivença/AM. Dos 10 municípios com maior população indígena, 5 estão no estado do Amazonas. Os dados dos 30 municípios com maior contingente populacional em terras e agrupamentos indígenas está na tabela 2. Nenhum deles possui UTI.

${ }^{3}$ A Terra Indígena Fulni-ô está localizada no município de Águas Belas e a sede do município está inserida na TI. Segundo os dados do Censo Demográfico de 2010, o total de pessoas indígenas residindo na TI era de 4.463 , ou seja, $18,7 \%$ da população total da TI, que era em 2010 de 23.882. Situação que difere da realidade dos demais municípios com maior população residente, apresentados nesta análise, como por exemplo o caso do município de Pacaraima, cuja sede fica inserida na TI São Marcos. Segundo os dados de 2010 o total de pessoas residentes na TI São Marcos era de 7.948 e o total de pessoas indígenas era de 6.162 , ou seja, $77,5 \%$ da população residente na TI. 
A partir da identificação dos polos de destino da população para acesso aos serviços de saúde, foi possível compará-los aos dados dos municípios de origem dos pacientes, podendo-se observar pontos de concentração da busca por assistência à saúde e também as áreas com maior quantidade de população dependente do destino, que podem ser prioritárias para a instalação de unidades emergenciais de saúde.

Nesse sentido, a figura 2 apresenta o contingente de população indígena nos municípios de origem dos pacientes em associação com os dados de população indígena total dependente das cidades de destino.

A tabela disponível no anexo, por sua vez, apresenta o grau de participação de cada município na população estimada residente em terras e agrupamentos indígenas que demandam ou podem vir a demandar por atendimento de saúde nos polos de atração.

Tabela 2: Municípios de origem dos fluxos para acesso a serviços de saúde com mais expressivos contingentes populacionais estimados de população residente em Terras e Agrupamentos Indígenas:

\begin{tabular}{|c|c|c|c|c|}
\hline UF & Município & Complexidade & $\begin{array}{l}\text { Estimativa de população residente em } \\
\text { TIs e agrupamentos (2019) }\end{array}$ & Leitos de UTI \\
\hline AM & SÃO GABRIEL DA CACHOEIRA & Não é polo & 44.208 & 0 \\
\hline PE & ÁGUAS BELAS ${ }^{4}$ & Não é polo & 33.774 & 0 \\
\hline PA & JACAREACANGA & Não é polo & 25.218 & 0 \\
\hline AM & TABATINGA & Baixa/média & 24.266 & 0 \\
\hline AM & SÃO PAULO DE OLIVENÇA & Não é polo & 22.639 & 0 \\
\hline AM & SANTA ISABEL DO RIO NEGRO & Não é polo & 20.140 & 0 \\
\hline MT & QUERÊNCIA & Não é polo & 16.770 & 0 \\
\hline AM & BENJAMIN CONSTANT & Não é polo & 15.577 & 0 \\
\hline MT & CAMPINÁPOLIS & Não é polo & 14.891 & 0 \\
\hline RR & PACARAIMA & Não é polo & 14.312 & 0 \\
\hline RR & ALTO ALEGRE & Não é polo & 14.249 & 0 \\
\hline RR & AMAJARI & Não é polo & 12.456 & 0 \\
\hline AM & BARCELOS & Não é polo & 12.372 & 0 \\
\hline AM & AUTAZES & Não é polo & 12.362 & 0 \\
\hline MT & FELIZ NATAL & Não é polo & 12.319 & 0 \\
\hline AM & ATALAIA DO NORTE & Não é polo & 12.247 & 0 \\
\hline RR & UIRAMUTÃ & Não é polo & 12.044 & 0 \\
\hline AM & BORBA & Não é polo & 10.555 & 0 \\
\hline PE & PESQUEIRA & Baixa/média & 10.403 & 0 \\
\hline RR & NORMANDIA & Não é polo & 10.017 & 0 \\
\hline PE & CARNAUBEIRA DA PENHA & Não é polo & 9.910 & 0 \\
\hline AM & SANTO ANTÔNIO DO IÇÁ & Baixa/média & 9.310 & 0 \\
\hline MG & SÃO JOÃO DAS MISSÕES & Não é polo & 8.969 & 0 \\
\hline PB & MARCAÇÃO & Não é polo & 8.827 & 0 \\
\hline RO & GUAJARÁ-MIRIM & Não é polo & 8.713 & 0 \\
\hline PA & ORIXIMINÁ & Não é polo & 8.680 & 0 \\
\hline AM & MAUÉS & Não é polo & 8.184 & 0 \\
\hline RO & NOVA MAMORÉ & Não é polo & 8.091 & 0 \\
\hline PA & SÃO FÉLIX DO XINGU & Não é polo & 8.073 & 0 \\
\hline
\end{tabular}


Figura 2: Estimativa de residentes em Terras e Agrupamentos Indígenas nos municípios de origem e destino da população para acesso a serviços de saúde

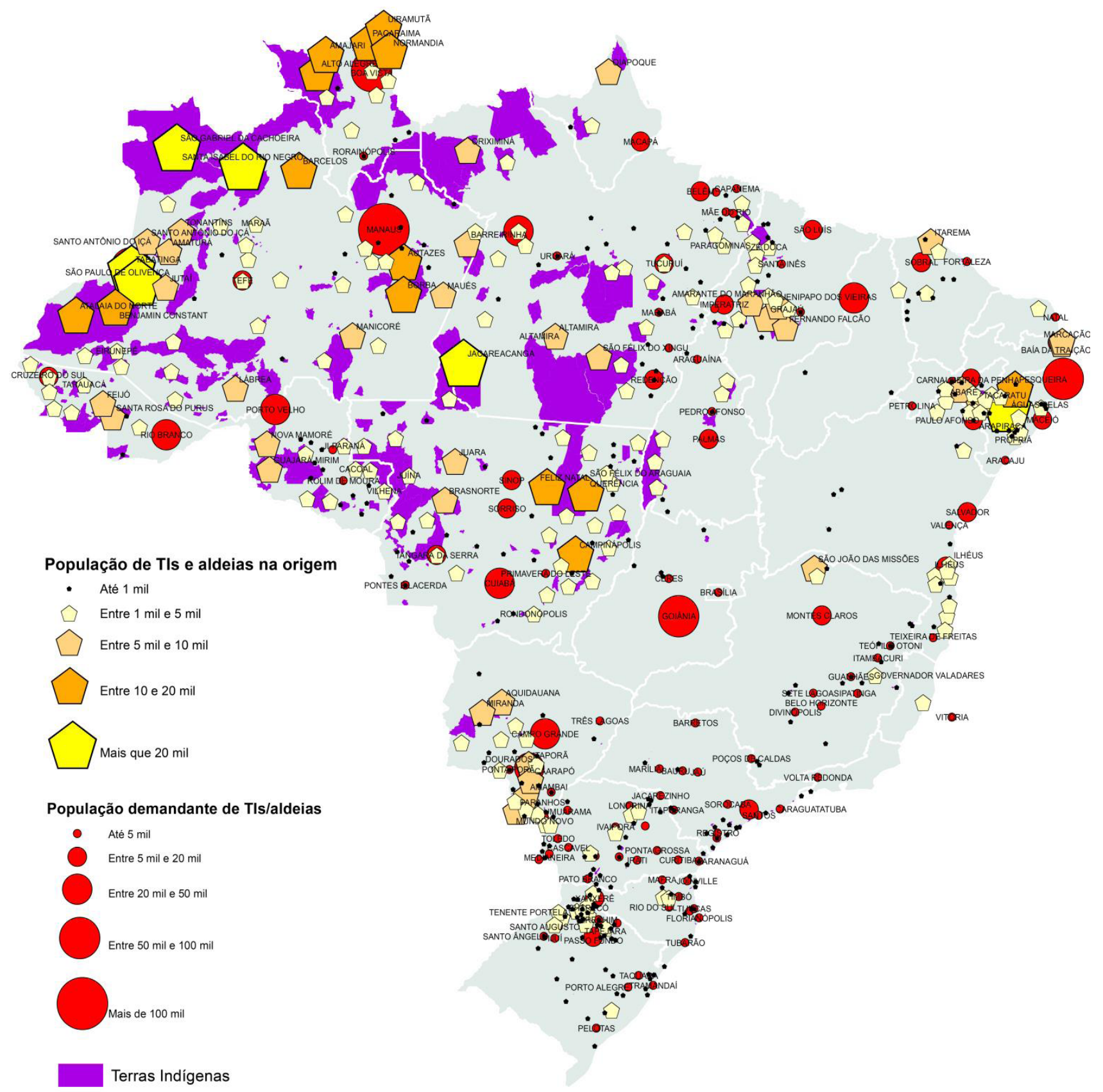

Fontes: Elaborado pelos autores, 2020. 
Os resultados apresentados permitem estabelecer um panorama dos deslocamentos da população indígena para atendimento à saúde e a participação de cada município de origem da população no volume total de população dependente dos municípios de destino, que são aqueles que teriam condições de efetuar o atendimento. A partir deste panorama, podese inferir que os municípios com maior participação no total de população dependente do município de destino podem ser considerados prioritários para a instalação emergencial de estruturas adicionais de atenção à saúde e/ou para serem alvos de investimentos que possam incrementar a capacidade de prestarem atendimentos de alta complexidade.

Nesse sentido, o principal desafio é buscar elementos que permitam a definição de municípios que, numa visão estratégica, poderiam ser alvos prioritários de investimentos e ações emergenciais que possam prestar o atendimento mais próximo à população indígena e, ao mesmo tempo, aliviar o volume de atendimentos de sistemas de saúde já sobrecarregados.

Essas conclusões partem inevitavelmente dos resultados que apresentamos anteriormente, mas buscaremos, nas linhas que se seguem, por enquadramentos regionais, desenvolvê-los de forma que a eles sejam adicionados elementos próprios das configurações territoriais locais, das redes de transporte e logística e da disponibilidade de receber grandes infraestruturas de saúde. Essa é uma avaliação preliminar dos autores e que necessariamente deverá ser articulada ao conhecimento das organizações indígenas, de lideranças comunitárias, outros especialistas, que poderão fornecer elementos adicionais para a tomada de decisão.

Partimos de dois critérios chave para a proposição de recomendações sobre municípios prioritários para o investimento em aumento de capacidade de atendimento de casos graves de COVID-19 entre a população indígena, são eles:

Critério 1: Municípios com percentual elevado de população indígena dependente de polos de alta complexidade localizados fora de seu município e com polos de média/baixa complexidade que atraem população de municípios com alto percentual de indígenas;

Critério 2: Municípios com percentual elevado de população indígena dependente de polos de alta e média/ baixa complexidade localizados fora de seu município e distantes de municípios polos de atendimento à saúde de alta ou média/baixa complexidade.
Além desses critérios, uma análise de contextualização dessas informações foi realizada para embasar a proposição de localização dos investimentos no atendimento a pacientes indígenas com sintomas graves de COVID-19. Essa análise levou em consideração a capacidade do local proposto servir de polo de atração da população indígena, em particular analisamos as principais vias e meios de acesso entre origem e destino e possibilidade de o polo atender a um número significativo de municípios com o qual estabelece relações.

Finalmente, foi ainda levada em consideração a hierarquização de total de óbitos por Unidades da Federação, considerando que os mesmos apontam para um total de casos de contaminação, considerando a dificuldade de notificação de todos os casos de contaminação, seja pela falta de sintomas, seja pela não realização de testagem em massa na população, e para a potencial saturação da rede de atendimento de alta complexidade.

Como é possível observar no Gráfico 1 e na tabela 3, as sete unidades da federação com maior número de óbitos são: São Paulo (3.416 pessoas); Rio de Janeiro (1.503); Ceará (966); Pernambuco (927); Amazonas (874); Pará (515) e Maranhão (330). Levando essas informações em consideração, esses estados assumiram uma prioridade maior nas recomendações. 
Gráfico 1 - Casos e óbitos de COVID-19 por UF de notificação

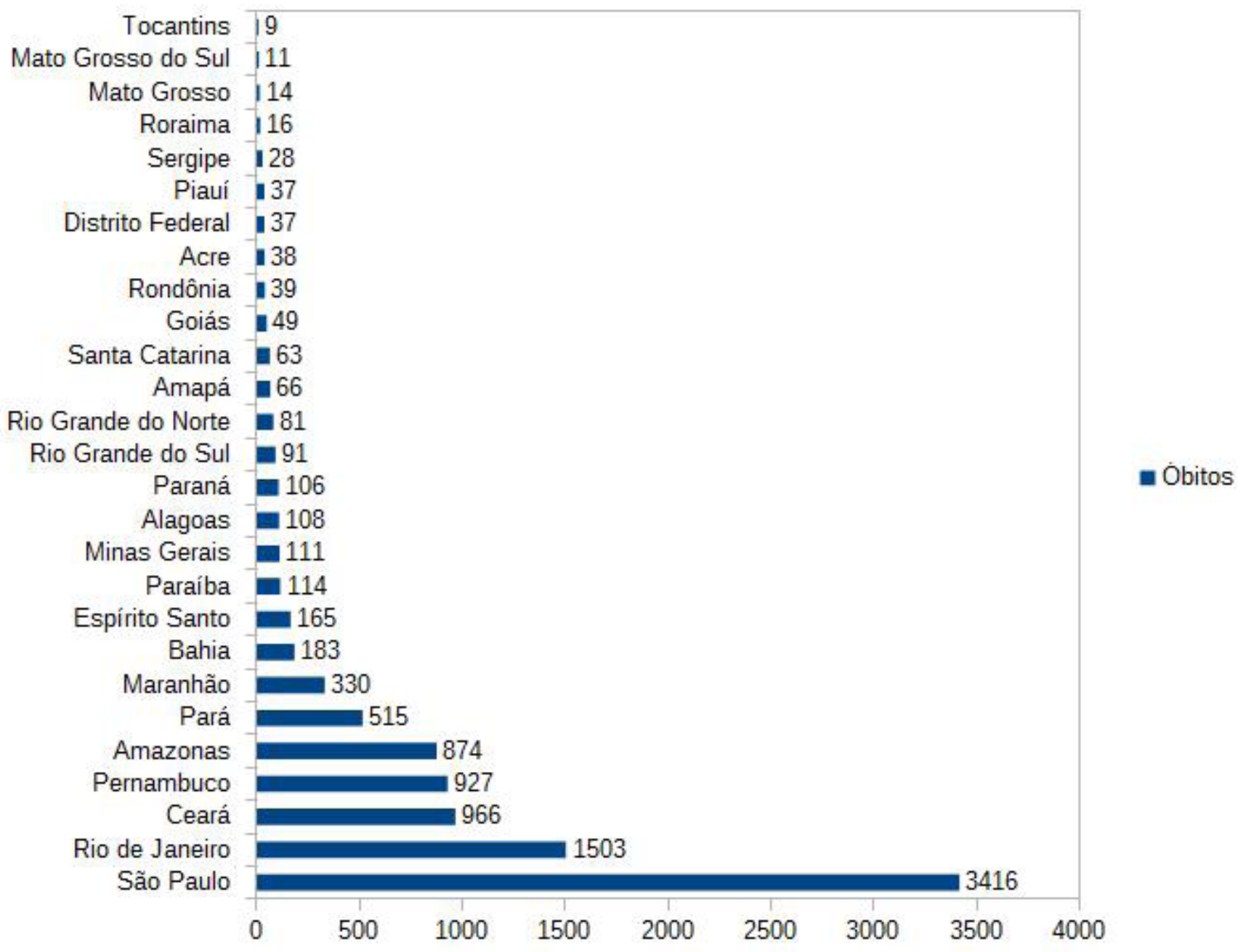

Fonte: Secretarias Estaduais de Saúde. Brasil, 2020 (https://covid.saude.gov.br/, Atualizado em: 08/05/2020, 19:00h) 


\begin{tabular}{|c|c|c|c|}
\hline Estado & Casos & Óbitos & $\begin{array}{l}\text { Taxa de } \\
\text { letalidade }\end{array}$ \\
\hline São Paulo & 41830 & 3416 & $8,17 \%$ \\
\hline Rio de Janeiro & 15741 & 1503 & $9,55 \%$ \\
\hline Ceará & 14956 & 966 & $6,46 \%$ \\
\hline Pernambuco & 11587 & 927 & $8,00 \%$ \\
\hline Amazonas & 10727 & 874 & $8,15 \%$ \\
\hline Pará & 6141 & 515 & $8,39 \%$ \\
\hline Maranhão & 5909 & 330 & $5,58 \%$ \\
\hline Bahia & 4818 & 183 & $3,80 \%$ \\
\hline Espírito Santo & 4242 & 165 & $3,89 \%$ \\
\hline Paraíba & 2031 & 114 & $5,61 \%$ \\
\hline Minas Gerais & 2943 & 111 & $3,77 \%$ \\
\hline Alagoas & 2033 & 108 & $5,31 \%$ \\
\hline Paraná & 1711 & 106 & $6,20 \%$ \\
\hline Rio Grande do Sul & 2447 & 91 & $3,72 \%$ \\
\hline Rio Grande do Norte & 1821 & 81 & $4,45 \%$ \\
\hline Amapá & 2322 & 66 & $2,84 \%$ \\
\hline Santa Catarina & 3205 & 63 & $1,97 \%$ \\
\hline Goiás & 1053 & 49 & $4,65 \%$ \\
\hline Rondônia & 1222 & 39 & $3,19 \%$ \\
\hline Acre & 1177 & 38 & $3,23 \%$ \\
\hline Distrito Federal & 2442 & 37 & $1,52 \%$ \\
\hline Piauí & 1131 & 37 & $3,27 \%$ \\
\hline Sergipe & 1438 & 28 & $1,95 \%$ \\
\hline Roraima & 1124 & 16 & $1,42 \%$ \\
\hline Mato Grosso & 457 & 14 & $3,06 \%$ \\
\hline Mato Grosso do Sul & 326 & 11 & $3,37 \%$ \\
\hline Tocantins & 494 & 9 & $1,82 \%$ \\
\hline
\end{tabular}


Manaus e a necessidade de novos polos no Alto Rio Negro, no Alto Solimões e em Itacoatiara

É importante destacar os municípios de São Gabriel da Cachoeira/AM, localizado a mais de $850 \mathrm{~km}$ de Manaus e somente acessível por barco ou avião, e Santa Isabel do Rio Negro/AM, a cerca de $630 \mathrm{~km}$ de Manaus, ambos localizados na região do Alto Rio Negro, que são juntos responsáveis por 64.348 indígenas e têm Manaus como único destino para acesso a serviços de saúde. Esses dois municípios são responsáveis por 33\% de toda a população indígena que têm Manaus como destino, não possuindo nenhum leito de UTI disponível.

Ainda no Amazonas, o município de Tabatinga/ AM, localizado a mais de $1.000 \mathrm{~km}$ de Manaus, polo de atendimentos de baixa/média complexidade, tem importância por atrair demandas dos municípios vizinhos e contribui com o volume de procura em Manaus, sendo responsável por $12 \%$ da demanda deste polo. Se somarmos o contingente centralizado em Tabatinga com aquele de São Gabriel da Cachoeira, temos um total de 84.038 pessoas dependentes de Manaus, o que representa $43 \%$ de toda a demanda desse polo.

Essas duas regiões - alto Rio Negro e alto Solimões - estão configuradas entre as cinco áreas mais dependentes do país, responsáveis juntas por um contingente de 148.386 indígenas, sendo as maiores pressões, dentre a população residente em terras e agrupamentos indígenas, sobre o sistema de saúde de Manaus, condição confirmada pelas características demográficas e certamente agravada pela situação geográfica, com ambas as cidades localizadas a distâncias expressivas de Manaus, com deslocamentos contados em dias de barco, e sem disponibilidade de leitos de UTIs. São, portanto, áreas que devem ser alvo de ações urgentes em termos da instalação de unidades adicionais de atendimento à saúde.

Além dos polos sugeridos em São Gabriel da Cachoeira e Tabatinga, que diminuiriam a pressão sobre Manaus de populações que ficam a distâncias significativas

Figura 3: Eixo Manaus-Parintins - Estimativa de residentes em terras e agrupamentos indígenas nos municípios de origem e destino da população para acesso a serviços de saúde

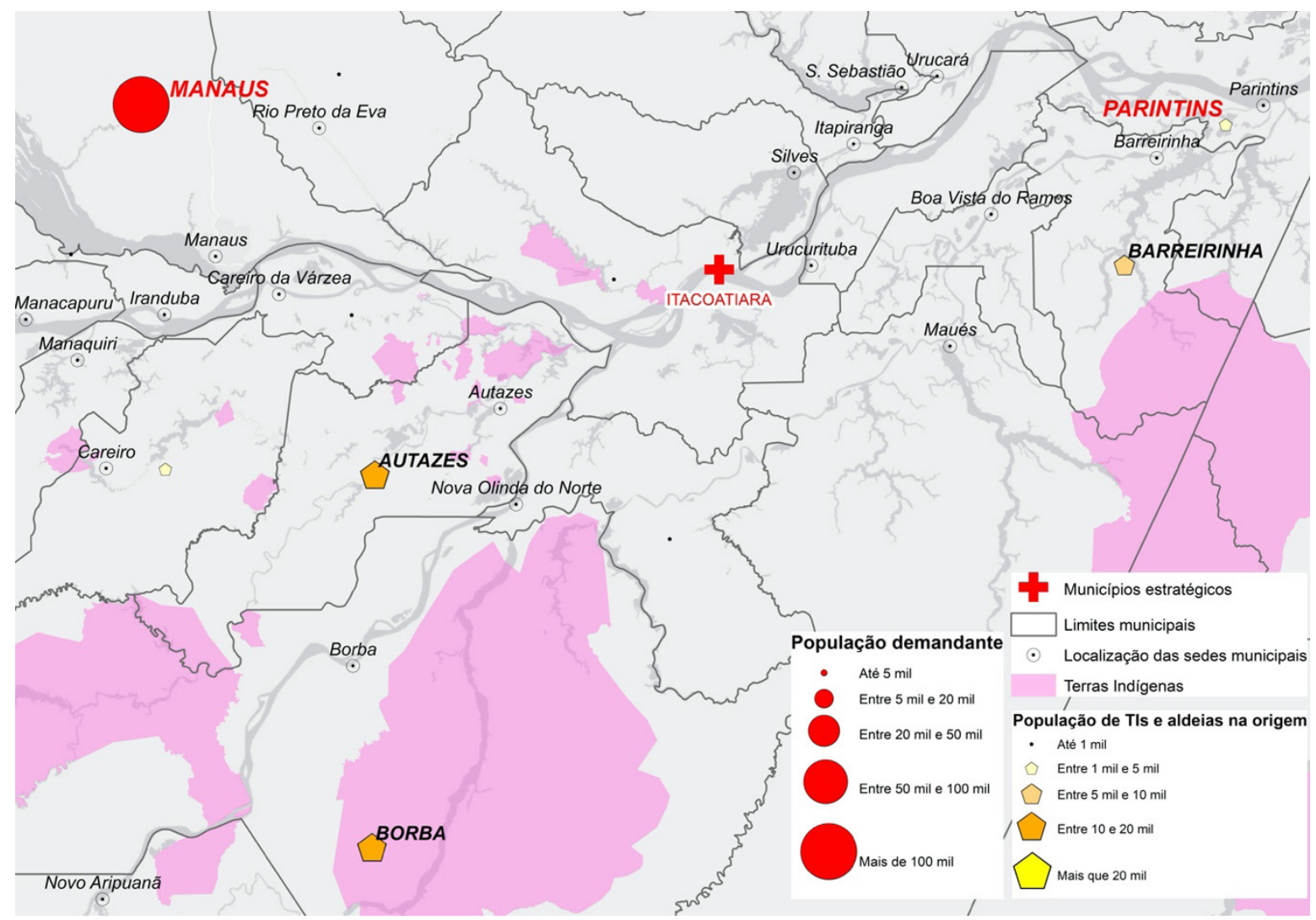

Fontes: Elaborado pelos autores, 2020. 
da capital, seria possível criar um polo emergencial no entorno de Manaus, que possibilitasse $o$ atendimento emergencial da população indígena dos municípios do eixo Parintins-Manaus (figura 3), com o potencial de atendimento de uma população indígena de $\mathbf{5 2 . 0 5 5}$ pessoas, incluindo a população indígena dependente do polo de baixa/média complexidade de Parintins (953).

Esse polo poderia reduzir 26,22 pontos percentuais da população indígena dependente de Manaus.

Esse polo precisaria do apoio de um barco para realização das remoções mais graves e ações preventivas na região, potencializando sua atração e o desafogamento de Manaus.

Considerando que a cidade de Itacoatiara tem acesso terrestre e fluvial, a sugestão é de colocação de um hospital de campanha nessa cidade para atendimento prioritário a 45.367 indígenas dos municípios de: Autazes (12.362); Barreirinha (7.993); Borba (10.555); Careiro da Várzea (840); Itacoatiara (971); Maués (8.184); Nova Olinda do Norte (137); Norte do Aripuanã (2.303); Nhamundá (953); Parintins (1.069); Urucará (1.070).

Com o potencial de atender ainda 5.618 indígenas de outros municípios próximos a Manaus que podem não conseguir vaga na capital, pelo alto número de casos e óbitos notificados no estado do Amazonas: Presidente Figueiredo (827); Rio Preto da Eva (143); Manaquiri (1.823); Manacapuru (1.176); Careiro (1.546); Iranduba (103).

Polo de Boa Vista: necessidade de fortalecimento e novo polo em Pacaraima/RR

Boa Vista é o polo de atendimento de alta complexidade de referência para municípios com alto percentual de população indígena, sendo polo de recepção de remoções aéreas do DSEI Yanomami, que atende a Terra Indígena Yanomami, e do DSEI Leste, que atende a região do Lavrado.

Contudo, Boa Vista possui apenas 17 leitos de UTI, para uma população dependente estimada desse polo de 75.450 indígenas. Sendo essa população apenas $7 \%$ da população demandante do polo de alta complexidade de Boa Vista. Nesse sentido, o reforço da capacidade de atendimento a pacientes indígenas com sintomas graves de COVID-19 em Boa Vista é premente.

A concentração de 26.356 indígenas nos municípios de Pacaraima (14.312) e Uiramutã (12.044) e a localização da cidade de Pacaraima, na fronteira com a Venezuela, com um conjunto de abrigos de indígenas e não-indígenas, com forte presença de agências das nações unidades voltadas para atendimento ao migrante e refugiado abrigados e do exército brasileiro, e com a recepção pelos indígenas da TI São Marcos de indígenas oriundos da Venezuela, torna o local particularmente interessante para a criação de um polo de atendimento. Este polo teria ainda a vantagem de diminuir a pressão sobre Boa Vista de $35 \%$ da demanda de atendimento a indígenas desta região.

Importante reforçar, contudo, que o município do Uiramutã possuiu uma dificuldade de acesso terrestre no período de chuvas que está prestes a iniciar, tornando o deslocamento mais demorado (entre 5 e 6 horas). Nesse sentido, é urgente reforçar a logística de transporte de pacientes do mesmo para Pacaraima, atendendo toda a região das serras, área de atuação do DSEI Leste.

Por outro lado, o município de Normandia está localizado na fronteira com a Guiana e também tem um fluxo intenso de indígenas que atravessam a fronteira. A população indígena de Normandia, estimada em 10.017 pessoas não teria como se deslocar para o polo de Pacaraima, sendo dependente de Boa Vista, mas precisaria de medidas de prevenção e de barreiras sanitárias para sua proteção. Essas barreiras sanitárias poderiam atender os dois municípios - Uiramutã e Normandia, se fossem instaladas na interligação da BR$433 \mathrm{com}$ a BR-174 (que liga Manaus e Venezuela) e na interligação da BR-433 com a BR-401 (que liga Boa Vista e Normandia).

Recife e a possibilidade de incrementar a centralidade de Paulo Afonso/BA

A cidade de Paulo Afonso está localizada no litoral Norte da Bahia e perto da divisa da Bahia com Pernambuco e Alagoas (figura 4).

Nesta cidade, servida de aeroporto comercial, existe um polo de baixa complexidade que já é destino dos municípios pernambucanos de Jatobá (4.213 indígenas) e Petrolândia (824) e do município de Água Branca (116) em Alagoas.

0 estado vizinho de Pernambuco é o quarto estado em total de óbitos por COVID-19 (927 pessoas) e conta com uma população indígena dependente de polos de alta complexidade em Recife (61.044) e Petrolina (1.707) e de baixa complexidade em Serra Talhada (12.663) e Salgueiro (584).

A transformação do polo de baixa complexidade de Paulo Afonso/BA em um polo capaz de atender casos graves da COVID-19 entre os indígenas permitiria atender toda a região do sertão do São Francisco e entorno, nessa região servida pelas $\mathrm{BR} 110,232$ e 316 , desafogando os polos de atendimento de alta complexidade em 
Figura 4: Pernambuco e Alagoas - Estimativa de residentes em terras e agrupamentos indígenas nos municípios de origem e destino da população para acesso a serviços de saúde

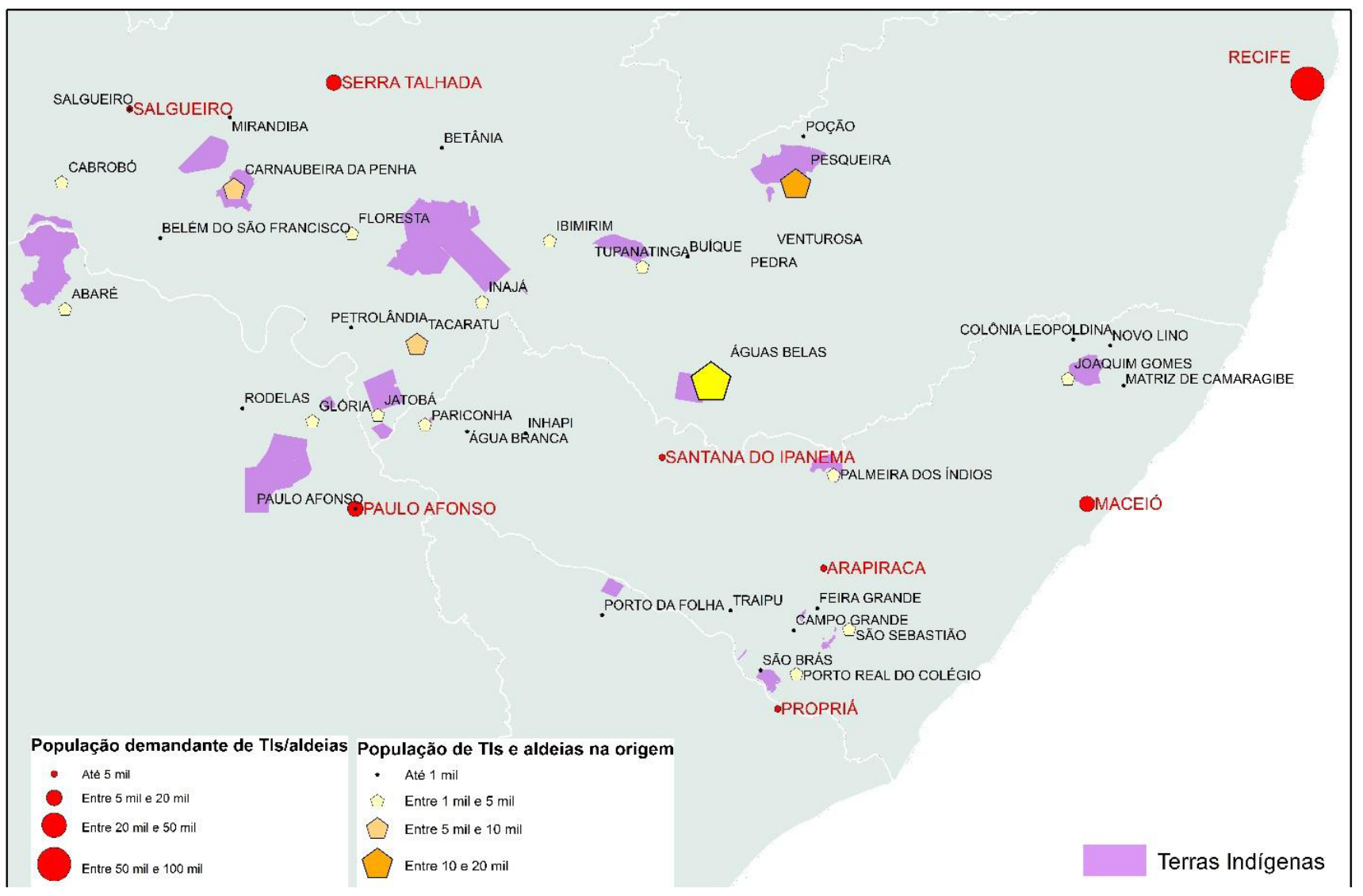

Fontes: Elaborado pelos autores, 2020.

Pernambuco, estado com alto índice de incidência da COVID-19.

O potencial de atendimento desse polo é de 87.027 indígenas, sendo:

- 75.998 de Pernambuco, dos municípios de Águas Belas (33.774), Pesqueira (10.403), Carnaubeira da Penha (9.910), Tacaratu (5.405), Jatobá (4.213), Cabrobó (4.041), Floresta (2.737), Ibimirim (2.358), Inajá (1.795), Tupanatinga (1.350), Petrolândia (824), Poção (721), Salgueiro (660), Buíque (536), Mirandiba (516), Orocó (104), Belém do São Francisco (68) e Betânia (17).

- 9.181 da Bahia dos municípios de Abaré (4.134), Glória (2843), Curaçá (1.603), Rodelas (476) e Paulo Afonso (124).

- 1.848 de Alagoas, dos municípios de Pariconha (1.616), Inhapi (116) e Água Branca (116).

Os demais municípios de Alagoas com população indígena, localizam-se mais perto de polos de alta complexidade com leitos de UTI, podendo ser atendidos nos mesmos se a curva de crescimento do número de casos não sofrer uma alteração forte nesse estado.
Embora Sergipe esteja com um total de casos e óbitos por COVID-19 notificados baixo, o polo de Paulo Afonso/BA poderia também atrair a população indígena do município de Porto da Folha (335), que fica a uma distância equivalente de Paulo Afonso e de Aracajú - seu atual polo de destino.

Goiânia e a necessidade de novos polos em Água Boa/ MT e Redenção/PA

O polo de Goiânia, de acordo com os dados produzidos, é responsável por acolher a população indígena de vários municípios localizados a mais de $500 \mathrm{~km}$ de distância da cidade (Querência, 848 km; Campinápolis, 595,6 km; São Felix do Xingu, 1.546 km; Nova Nazaré, 518 km; São Félix do Araguaia, 805 km; Canarana, 709 km e Ribeirão Cascalheira, $763 \mathrm{~km}$ ), conforme pode ser observado na figura 5 .

Todos esses municípios ficam no eixo da BR-158, no trecho que liga Barra do Garças, no Mato Grosso, a 
Figura 5: Municípios dependentes de Goiânia, no eixo da BR-158 - Estimativa de residentes em Terras e A grupamentos Indígenas nos municípios de origem e destino da população para acesso a serviços de saúde

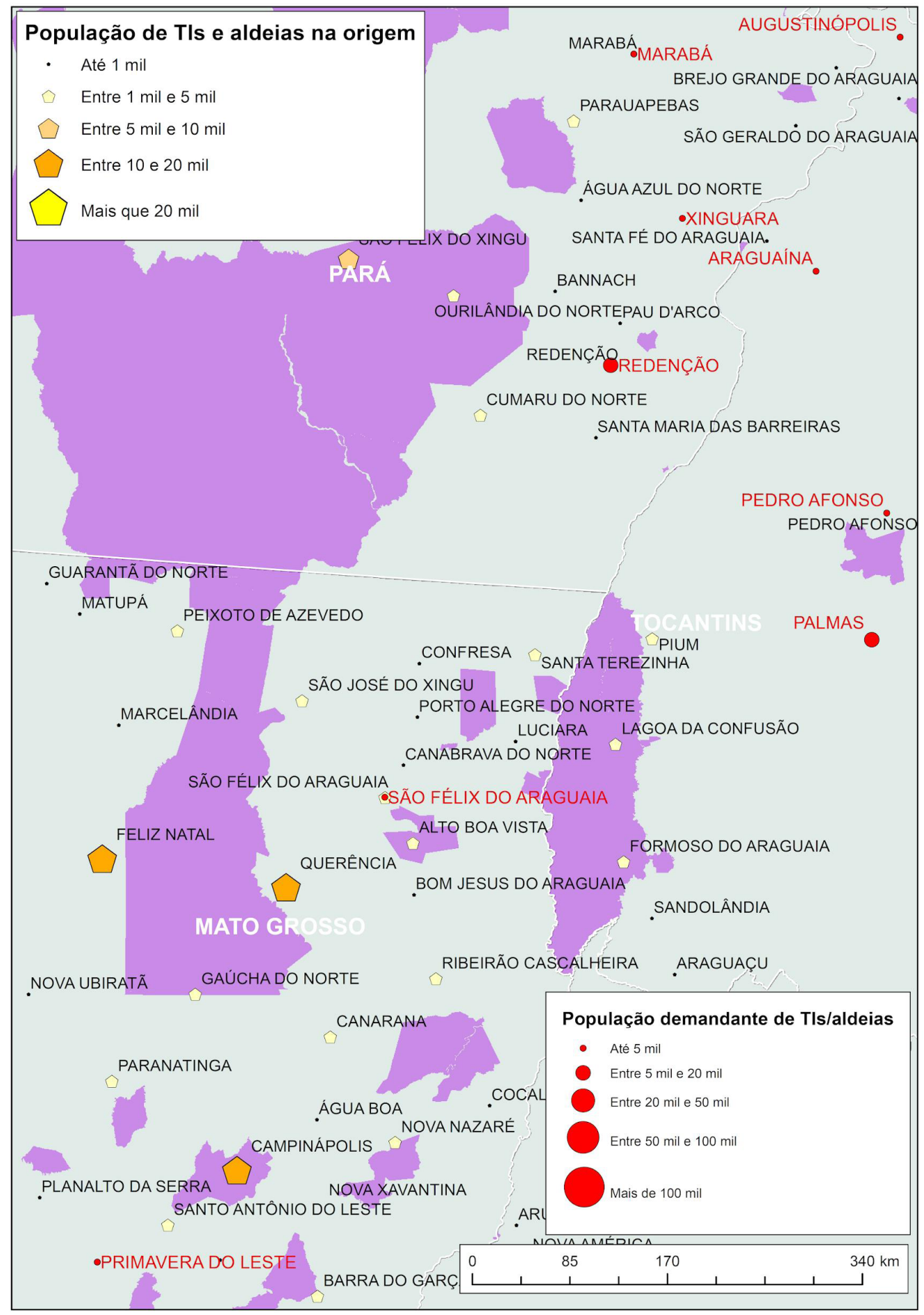

Fontes: Elaborado pelos autores, 2020.

Redenção, no Pará. Juntos, esses municípios respondem por uma população indígena de 48.891 pessoas.

Goiânia também é referência para toda a população vinda dos municípios que tem como destino Redenção/ PA, que sozinha tem uma população dependente de 6.404 pessoas, atendendo os municípios de Ourilândia do Norte, Cumaru do Norte, Pau d'Arco e Bannach.
Por isso, seria muito importante a descentralização de serviços nesta região, podendo compreender um polo em Água Boa, cidade matogrossense com 25.721 habitantes, de acordo com as últimas estimativas do IBGE, e com posição central em relação a São Félix do Araguaia, Campinápolis, Nova Nazaré e Ribeirão da Cascalheira, que poderia ser nova referência para 45.778 
indígenas.

Outro polo de especial importância estaria em Redenção, no Pará, cidade que abriga a sede do Distrito Especial de Saúde Indígena (DSEI) Kaiapó do Pará, que seria responsável para servir de referência para uma população total de 14.477 indígenas.

\section{Dourados/MS e a necessidade de estruturação em Amambai/MS}

A população indígena dos municípios situados na chamada região do cone sul do Mato Grosso do Sul que são atendidos em Dourados chega a 38 mil pessoas (figura 6). Depois de Dourados o polo mais próximo onde existe atendimento de alta complexidade é a capital do estado, Campo Grande situada a cerca de 4 horas de viagem ao norte. A capacidade de Campo Grande é de 106 leitos de UTI; Dourados possui 46 leitos de UTI e a população indígena que é dependente de Dourados é de 38 mil pessoas como apontado acima. Campo Grande atende toda a região ao norte da capital, que tem a população indígena estimada em cerca de 30 mil pessoas.

Em Dourados a população indígena dependente oriunda de outros municípios é maior do que aquela que de sai de Dourados para ser atendida na cidade de Campo Grande. Com isso o polo de Dourados estará com a capacidade lotada e fará pressão para o atendimento na capital também. Já Amambai, que possuiu $21 \%$ da população dependente de Dourados, com uma localização mais cerca das terras e agrupamentos indígenas poderia atender essa população e ainda as terras e os agrupamentos indígenas do noroeste do Paraná, fronteira com essa região do Mato Grosso do Sul.

Figura 6: Dourados/MS - Estimativa de residentes em Terras e Agrupamentos Indígenas nos municípios de origem e destino da população para acesso a serviços de saúde

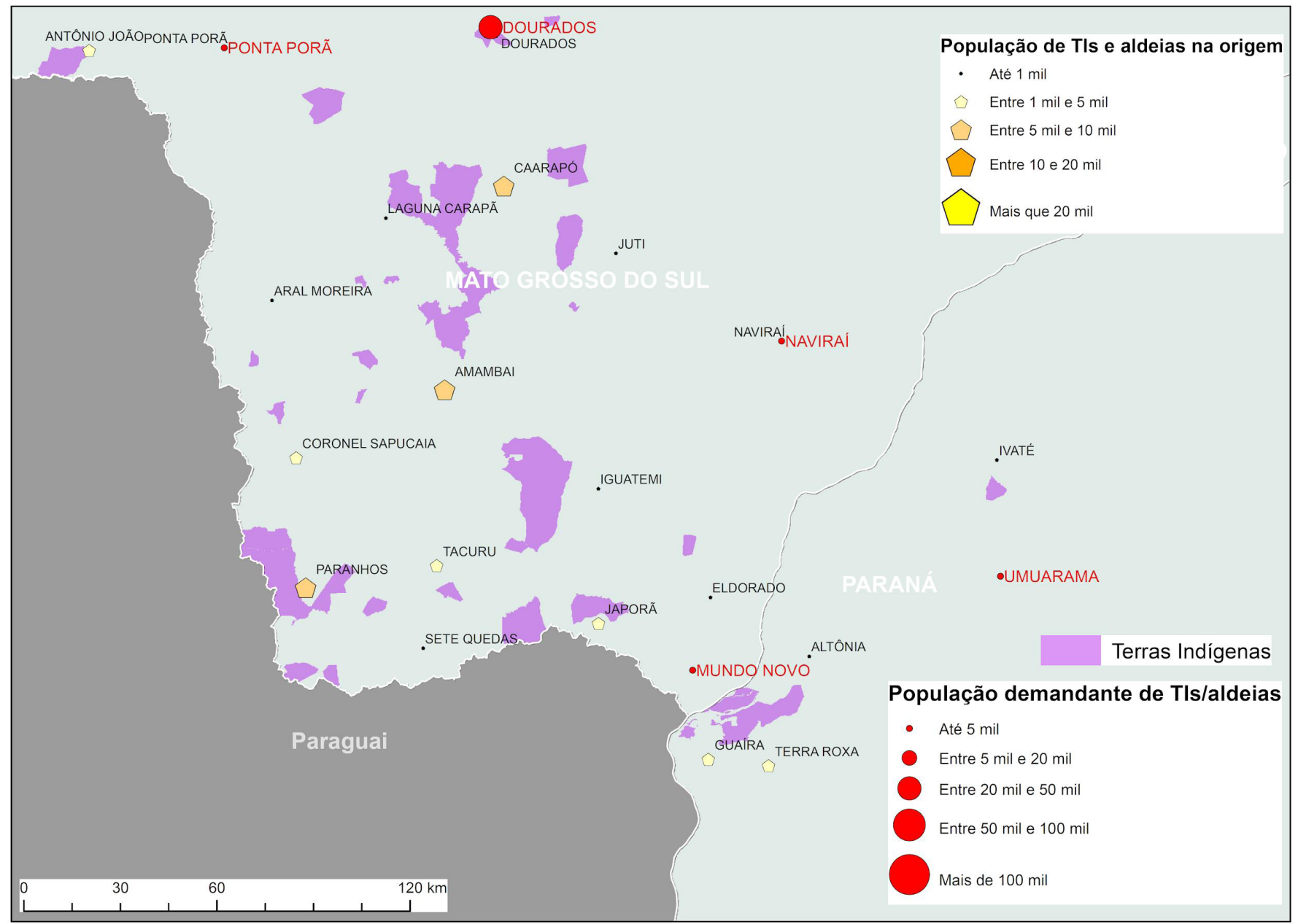

Fontes: Elaborado pelos autores, 2020. 
Polo de Santarém/PA: dificuldades logísticas em Jacareacanga/PA e Oriximiná/PA

O polo de Santarém é responsável pela recepção de 41.100 indígenas, provenientes de 7 municípios: Jacareacanga (25.218), Oriximiná (8.680), Aveiro (3.008), Itaituba (1.488), Óbidos (1.237), Belterra (1.156) e Trairão (315).

Com uma área de aproximadamente $53.304 \mathrm{~km}^{2}$, maior que o estado do Rio de Janeiro, localizado na região do Tapajós, o município de Jacareacanga dista aproximadamente $758 \mathrm{~km}$ de Santarém, em trecho em sua maior parte não pavimentado da Rodovia Transamazônica (BR-230). Outra opção mais rápida é o transporte aéreo. Por isso, devido ao grande contingente populacional da cidade, é certamente um polo para a instalação de estruturas emergenciais de atendimento à saúde indígena.

Por outro lado, o município de Oriximiná é apontado como outro polo estratégico devido à característica dispersa das ocupações, estando a população de mais de 10 mil indígenas localizada em 58 pequenas localidades (IBGE, 2020a) ao longo de furos e lagos amazônicos ao longo dos mais de $135 \mathrm{mil} \mathrm{km}^{2}$ que contemplam a área dos municípios vizinhos de óbidos e Oriximiná somados, o que equivale a três vezes a área do estado do Rio de Janeiro. Nessas áreas, será fundamental a descentralização dos recursos de saúde a partir de Santarém. Esses municípios somados também possuem 71 localidades quilombolas.

\section{A centralidade de Teresina/PI e a necessidade de novos polos em Altamira/PA e Grajaú/MA}

O polo de Teresina apresenta uma população dependente de 33.928 indígenas, a contribuição de um conjunto de municípios do estado do Maranhão e do Pará que acessam a cidade através da BR-226 e pela

Figura 7: Teresina/PI - Estimativa de residentes em Terras e Agrupamentos Indígenas nos municípios de origem e destino da população para acesso a serviços de saúde

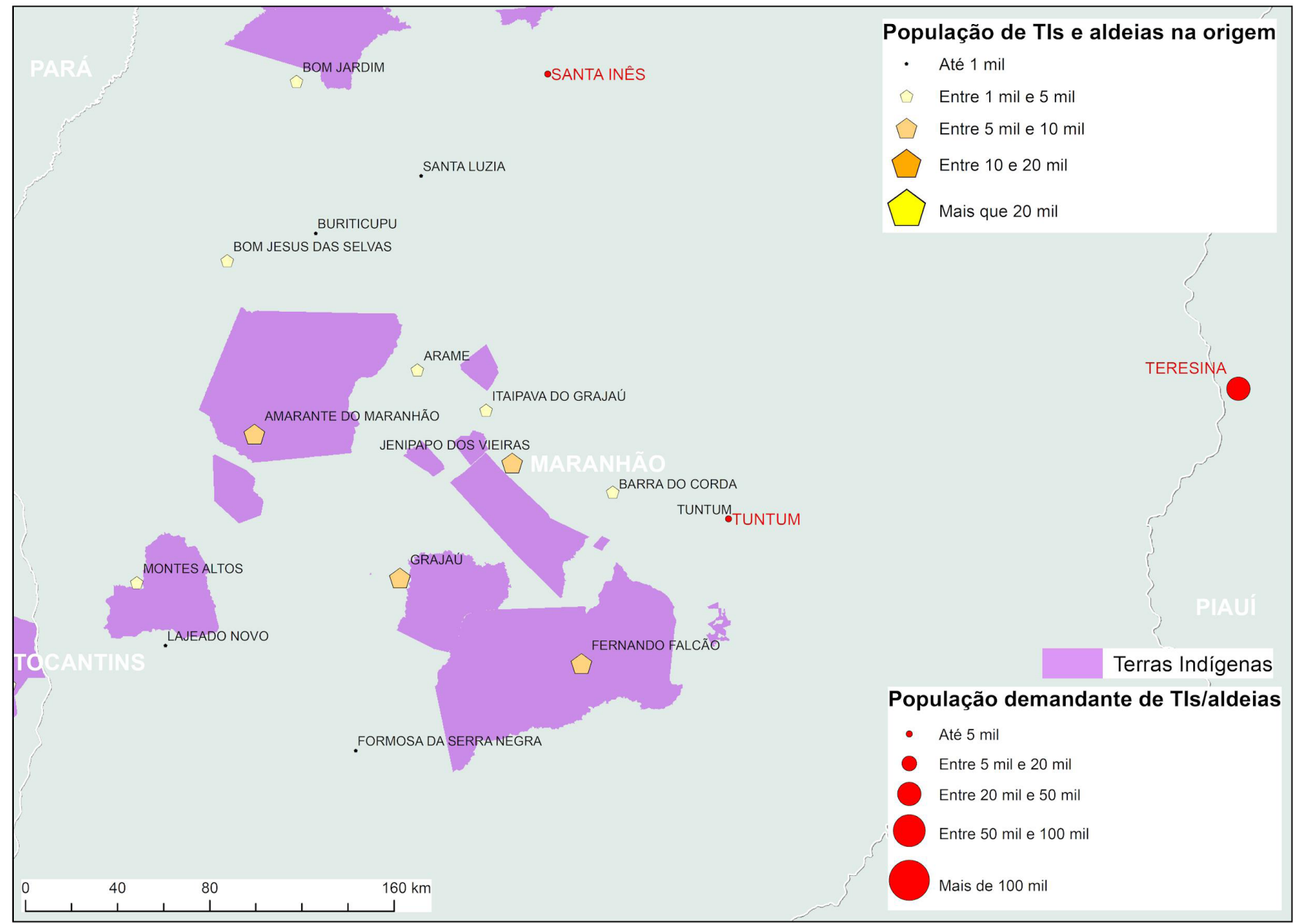

Fontes: Elaborado pelos autores, 2020. 
Rodovia Transamazônica (BR-230): Altamira, no Pará (6.117), e, do Maranhão, Grajaú (6.109), Fernando Falcão (5.563), Jenipapo dos Vieiras (5.332), Barra do Corda (4.829), Arame (2.246) e outros 5 municípios (figura 7).

Esses dados mostram a importância de investimentos para que a população dependente oriunda de Altamira tenha atendimento no próprio município, evitando-se assim o deslocamento de aproximadamente $1.650 \mathrm{~km}$ pela Transamazônica até Teresina, em um percurso que pode levar mais de 22 horas de viagem.

A cidade de Grajaú também necessitaria ser alvo de investimentos na constituição de um novo polo, capaz de atender a população indígena proveniente dos municípios de Fernando Falcão, Jenipapo dos Vieiras, Barra do Corda, Arame, Montes Altos, Tuntum, Buriticupu, Lajeado Novo e Formosa da Serra Negra. Esse polo poderia absorver um contingente de 27.345 pessoas provenientes desses municípios.

\section{Porto Velho: Guarajá-Mirim/RO}

0 polo de Porto Velho apresenta a população dependente de 30.789 indígenas, sendo 16.804 , isto é, $54 \%$ do total, proveniente dos municípios de GuajaráMirim e Nova Mamoré, de modo que sugerimos que sejam pensadas soluções que contemplem a realização de investimentos para atenção à saúde da população indígena proveniente destes dois municípios.

Polo de Rio Branco: o isolamento de Santa Rosa do Purus/AC e Tarauacá/AC

O polo de Rio Branco atrai 26.836 indígenas, que dependem do mesmo para atendimento de alta complexidade, recebendo parte da população atendida no polo de alta complexidade do Cruzeiro do Sul e do polo de baixa/média complexidade de Tarauacá, além dos municípios de Boca do Acre/AM e Pauini/AM (figura

Figura 8: Estado do Acre - Estimativa de residentes em Terras e Agrupamentos Indígenas nos municípios de origem e destino da população para acesso a serviços de saúde

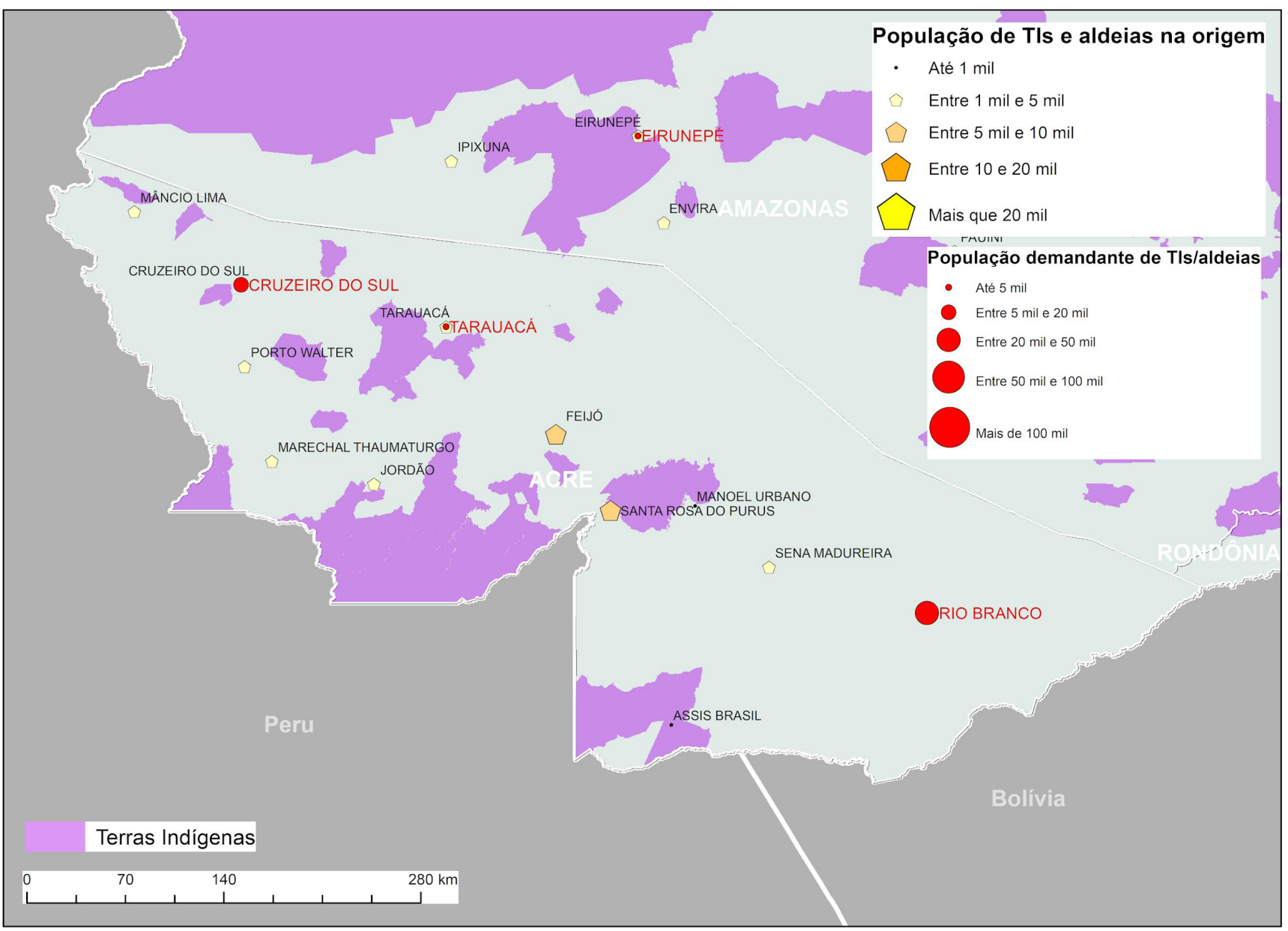


8)

Dentre os municípios com população indígena dependente de Rio Branco, temos o município de Santa Rosa do Purus, que tem sua população indígena estimada em 6.950 pessoas. Em caso de necessidade, os pacientes em estado grave são removidos de táxiaéreo ou helicóptero para Rio Branco. A outra forma de transporte para acesso à saúde é o uso do transporte fluvial até Manoel Urbano, uma viagem longa, e depois pela BR-364 para Rio Branco.

A região dos municípios de Jordão, Tarauacá, Feijó e Santa Rosa do Purus (19.754 indígenas no total) possui algumas terras indígenas com povos isolados e de recente contato e está localizada numa região de fronteira com o Perú, onde se localizam as reservas indígenas peruanas, tornando-se uma região de possível trânsito transfronteiriço de população indígena que pode ampliar o contágio e/ou a demanda por atendimento de saúde na parte brasileira.

Nessa região seria importante transformar o polo de baixa e média complexidade de Tarauacá num polo capaz de atender casos mais graves de COVID-19 entre os povos indígenas, considerando sua centralidade entre Cruzeiro do Sul e Rio Branco, quando o deslocamento for terrestre pela BR-364, assim como a possibilidade de deslocamento terrestre a partir de cidades como Feijó, Manoel Urbano e Sena Madureira que recebem a população residente nos braços dos rios. Esse polo teria o potencial de desafogar a demanda de $40 \%$ da população indígena dependente de Rio Branco, ao redirecioná-la para Tarauacá no caso de suspeita de COVID-19 e da presença de sintomas graves não tratáveis pelos postos de saúde, polos base de saúde indígena ou agentes indígenas de saúde.

O polo de Tarauacá poderia atrair uma população indígena de 15.012 indígenas dos municípios de: Feijó (5.525); Tarauacá (2.987); Sena Madureira (1.563); Manoel Urbano (645); e Jordão (4.293).

Por outro lado, o isolamento de Santa Rosa do Purús, torna o município particularmente importante para ações preventivas e de criação de barreiras sanitárias, considerando que já foram reportados casos suspeitos de COVID-19 na cidade e a dificuldade de acesso e transporte da região.

\section{Oiapoque/AP}

O município de Oiapoque, localizado no extremo norte do estado do Amapá, é responsável por uma população dependente de 5.690 indígenas do total de 7.265 que acorrem a Macapá, o que representa $78,3 \%$ do total.
Além disso, a cidade está localizada a cerca de $580 \mathrm{~km}$ da capital, em trajeto realizado por estrada em sua maior parte não pavimentada, numa viagem que pode durar entre 10 e 12 horas.

Por isso, sinalizamos a importância de que o Oiapoque seja considerado como um polo para a instalação de estruturas emergenciais de atenção à saúde indígena.

\section{Polo em Itarema/CE}

O Ceará encontra-se em terceiro lugar em total de pessoas cujo óbito foi notificado associado à COVID-19 e quando analisamos os dados associados à figura 2 é possível verificar que existe uma população indígena dependente dos polos de Sobral (12.476 indígenas) e Fortaleza (3.111) que representa $1 \%$ e $0,3 \%$ do total de pessoas que dependem desses polos, nesse estado onde o sistema de saúde está no limite de sua capacidade de resposta e o sistema de lockdown já foi implementado na cidade de Fortaleza.

A recomendação é de que seja instalado um hospital de campanha no município de Itarema onde a população indígena éresponsável por $62 \%$ da população dependente de Sobral. Esse município poderia servir de polo para a população indígena dos municípios que dependem de Sobral - Monsenhor Tabosa (2.249 indígenas), Poranga (2.050), Tamboril (311), Acaraú (108); assim como dos municípios que dependem de Fortaleza - Itapipoca (1.367), Crateús (750), Aratuba (389), Quiterianópolis (311), Novo Oriente (145), Boa Viagem (75) e Canindé (75).

O polo de Itarema/CE poderia ser referência para o atendimento de 15.587 indígenas.

\section{Recomendações}

Considerandoadiscussãoderesultadosapresentados acima, recomendamos a criação e/ou reforço de ao menos 17 polos de atendimento emergencial e preventivo aos povos indígenas para enfrentamento da COVID-19 nos municípios de São Gabriel da Cachoeira/ AM, Tabatinga/AM, Itacoatiara/AM, Pacaraima/RR, Boa Vista/RR, Paulo Afonso/BA, Água Boa/MT, Redenção/ $\mathrm{PA}$, Jacareacanga/PA, Oriximiná/PA, Amambai/MS, Grajaú/MA, Altamira/PA, Guajará-Mirim/RO, Tarauacá/ $\mathrm{AC}$, Oiapoque/AP e Itarema/CE, que representamos no cartograma da figura 9. 


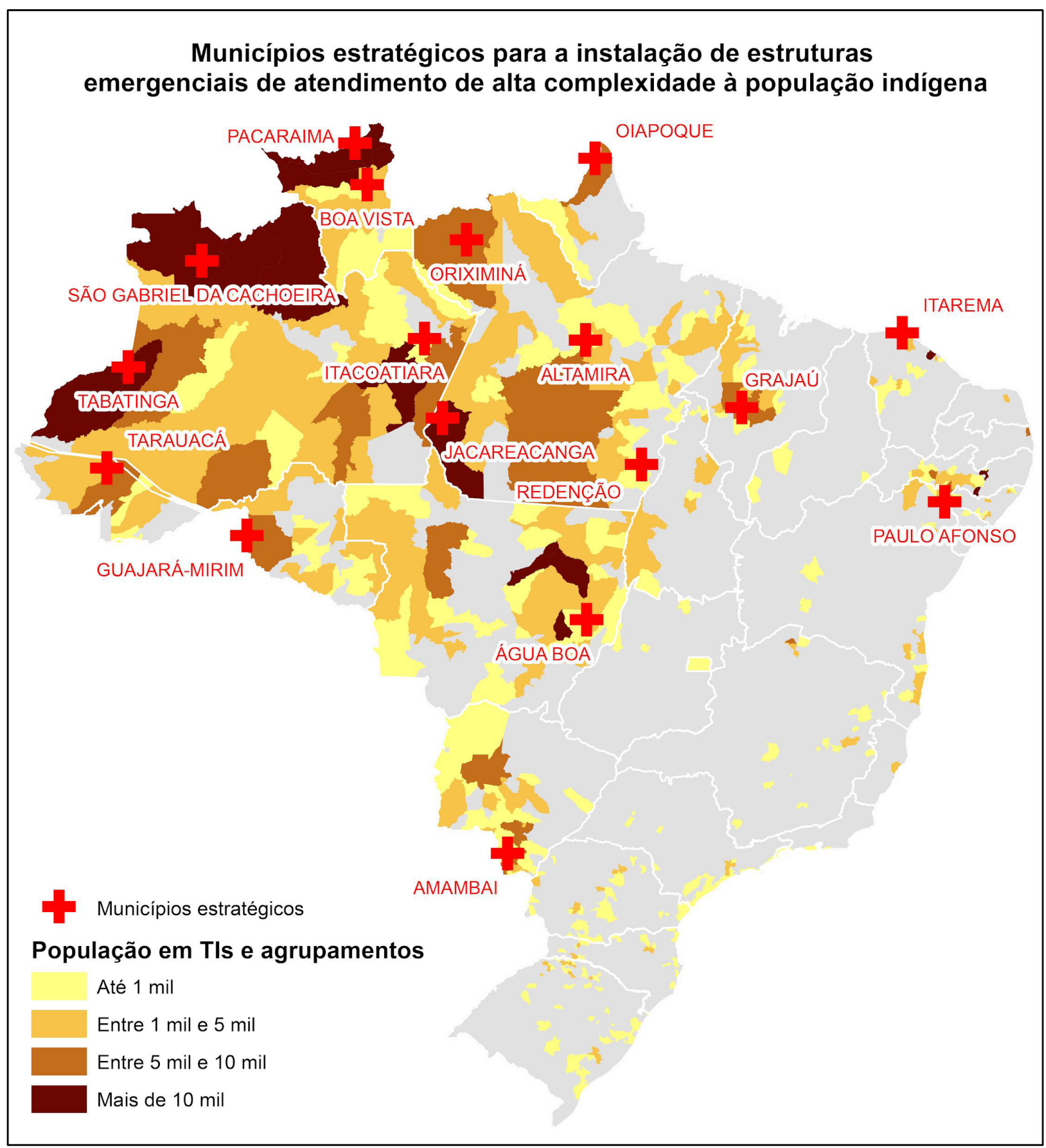

Fontes: Elaborado pelos autores, 2020.

Agradecimentos

Agradecemos as contribuições de Lara Torchi Esteves, José Nagib da Silva Lima e Eli Marta Veras Barroso, fundamentais para maior conhecimento das realidades locais do Acre, de Roraima e do Maranhão, respectivamente. 


\section{Referências}

AZEVEDO, Marta; DAMASCO, Fernando; ANTUNES, Marta; MARTINS, Marcos Henrique; REBOUÇAS, Matheus Pinto. Análise de Vulnerabilidade Demográfica e Infraestruturaldas Terras Indígenas à Covid-19 - Caderno de insumos. Disponível em: https://www.nepo.unicamp.br/publicacoes/Caderno-Demografia-Indigena-e-COVID19.pdf, acesso em 09 mai2020.

COE-COVID19/MS. Boletim Epidemiológico Especial n 15. Ministério da Saúde. Secretaria de Vigilância em Saúde. Atualizado em 08/05/2020, disponível em: https://portalarquivos.saude.gov.br/images/pdf/2020/May/09/2020-0506-BEE15-Boletim-do-COE.pdf

IBGE. Base de Informações sobre os Indígenas e Quilombolas 2019. Rio de Janeiro: IBGE, 2020a. Disponível em: www.ibge.gov.br/geociencias/organizacao-do-territorio/tipologias-do-territorio/27480-base-de-informacoes-sobre-ospovos-indigenas-e-quilombolas. $h t m l ?=\& \mathrm{t}=0$-que-e, acesso em 06mai2020.

Regiões de Influência das Cidades - REGIC 2018. Rio de Janeiro: IBGE, 2020b. Disponível em: https://www.ibge. gov.br/geociencias/organizacao-do-territorio/redes-e-fluxos-geograficos/15798-regioes-de-influencia-das-cidades. html?=\&t=o-que-e, acesso em 06mai2020.

O IBGE apoiando o combate à Covid-19. Disponível em: https://covid19.ibge.gov.br/, acesso em 09mai2020c.

Arranjos populacionais e concentrações urbanas no Brasil. 2 ed. Rio de Janeiro: IBGE, 2016. 167 p.

. Censo Demográfico 2010. Características gerais dos indígenas. Resultados do universo. Rio de Janeiro: IBGE. 2012, 245p.

MINISTÉRIO DA SAÚDE. O SUS de A a Z: garantindo saúde nos municípios. 3 ed. Brasília: Ed. do Ministério da Saúde, 2009.Disponívelem:https://bvsms.saude.gov.br/bvs/publicacoes/sus_az_garantindo_saude_municipios_3ed_p1.pdf. Acesso em 29 out. 2019.

MINISTÉRIO DA SAÚDE. Coronavírus Brasil. Disponível em: https://covid.saude.gov.br/, acesso em 09mai2020.

NÚCLEO de Métodos Analíticos para Vigilância Epidemiológica do PROCC/Fiocruz \& GRUPO de Trabalho sobre Vulnerabilidade Sociodemográfica e Epidemiológica dos Povos Indígenas no Brasil à Pandemia de COVID-19. Risco de espalhamento da COVID-19 em populações indígenas: considerações preliminares sobre vulnerabilidade geográfica e sociodemográfica. Disponível em:

http://gitlab.procc.fiocruz.br/mave/repo/blob/master/Relatórios\%20técnicos\%20-\%20COVID-19/procc-emap-enspcovid-19-report4_20200419-indigenas.pdf, acesso em 09mai2020. 
Anexo

Tabela 4: Participação dos municípios de origem no volume de população dependente dos polos de atração (destino) da população estimada residente em Terras e Agrupamentos Indígenas para acesso a serviços de saúde (2019)

Município (polo) de destino

\section{MANAUS}

Estimativa de população dependente em TIs e agrupamentos

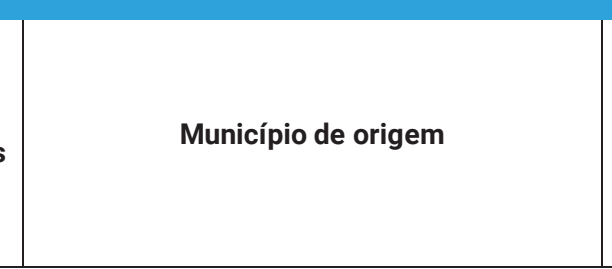

194.885
Estimativa populacional em

Tls e agrupamentos
Participação na população total dependente do destino

\begin{tabular}{|c|c|c|}
\hline SÃO GABRIEL DA CACHOEIRA & 44.208 & $23 \%$ \\
\hline TABATINGA & 24.266 & $12 \%$ \\
\hline SANTA ISABEL DO RIO NEGRO & 20.140 & $10 \%$ \\
\hline BARCELOS & 12.372 & $6 \%$ \\
\hline AUTAZES & 12.362 & $6 \%$ \\
\hline BORBA & 10.555 & $5 \%$ \\
\hline MAUÉS & 8.184 & $4 \%$ \\
\hline BARREIRINHA & 7.993 & $4 \%$ \\
\hline LÁBREA & 7.657 & $4 \%$ \\
\hline JUTAÍ & 6.536 & $3 \%$ \\
\hline MANICORÉ & 5.613 & $3 \%$ \\
\hline TAPAUÁ & 3.511 & $2 \%$ \\
\hline EIRUNEPÉ & 3.249 & $2 \%$ \\
\hline TEFÉ & 2.715 & $1 \%$ \\
\hline IPIXUNA & 2.404 & $1 \%$ \\
\hline NOVO ARIPUANÃ & 2.303 & $1 \%$ \\
\hline BERURI & 2.068 & $1 \%$ \\
\hline MANAQUIRI & 1.823 & $1 \%$ \\
\hline UARINI & 1.701 & $1 \%$ \\
\hline ITAMARATI & 1.647 & $1 \%$ \\
\hline NOVO AIRÃO & 1.596 & $1 \%$ \\
\hline CAREIRO & 1.546 & $1 \%$ \\
\hline COARI & 1.476 & $1 \%$ \\
\hline MANACAPURU & 1.176 & $1 \%$ \\
\hline URUCARÁ & 1.070 & $1 \%$ \\
\hline PARINTINS & 1.069 & $1 \%$ \\
\hline ANAMÃ & 979 & $1 \%$ \\
\hline ITACOATIARA & 971 & $0 \%$ \\
\hline ANORI & 890 & $0 \%$ \\
\hline CAREIRO DA VÁRZEA & 840 & $0 \%$ \\
\hline PRESIDENTE FIGUEIREDO & 827 & $0 \%$ \\
\hline CARAUARI & 438 & $0 \%$ \\
\hline CANUTAMA & 316 & $0 \%$ \\
\hline RIO PRETO DA EVA & 143 & $0 \%$ \\
\hline NOVA OLINDA DO NORTE & 137 & $0 \%$ \\
\hline IRANDUBA & 103 & $0 \%$ \\
\hline PACARAIMA & 14.312 & $19 \%$ \\
\hline
\end{tabular}




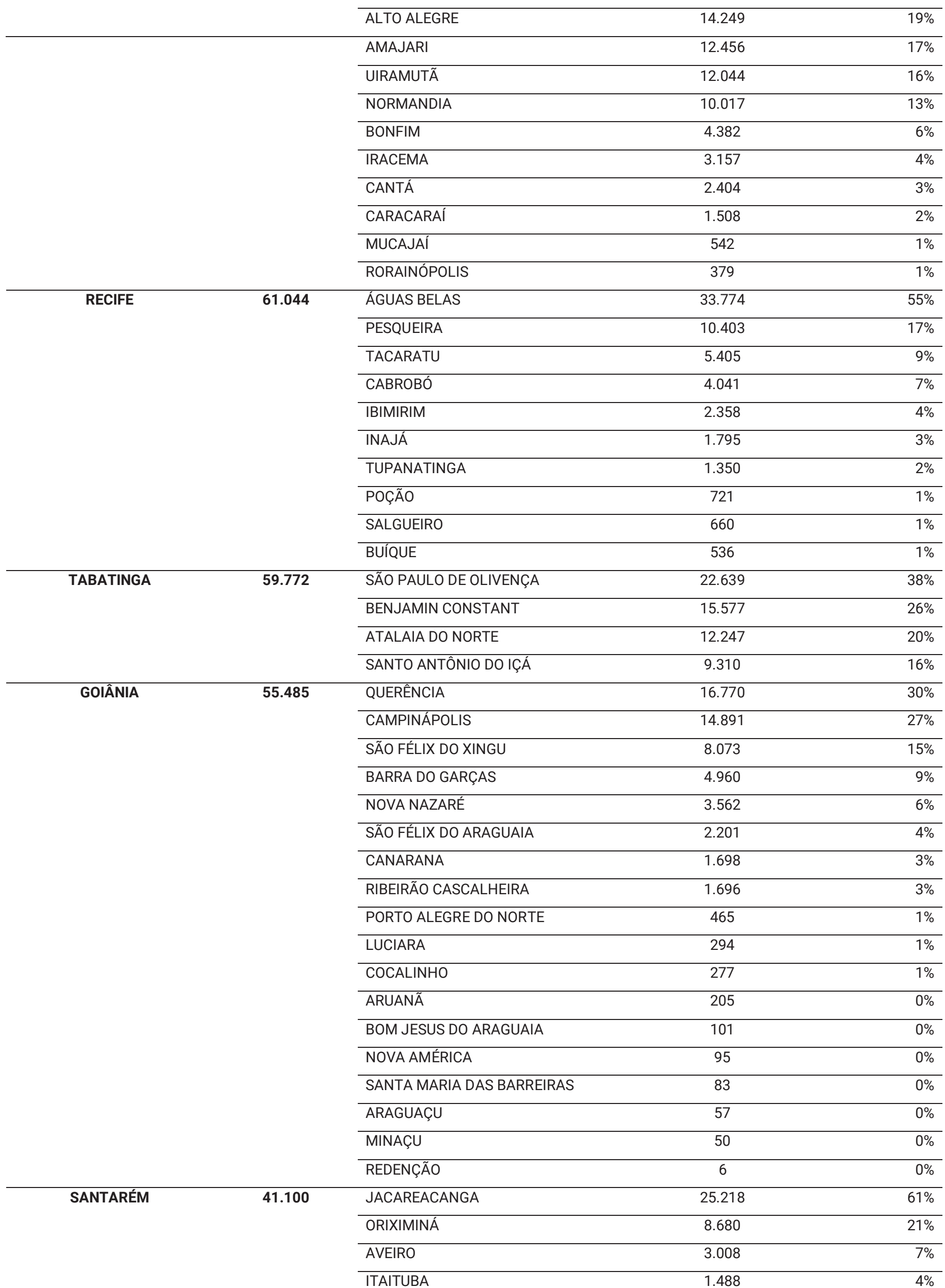




\begin{tabular}{|c|c|c|c|c|}
\hline & & & & \\
\hline & & ÓBIDOS & 1.237 & $3 \%$ \\
\hline & & BELTERRA & 1.156 & $3 \%$ \\
\hline & & TRAIRÃO & 315 & $1 \%$ \\
\hline \multirow[t]{16}{*}{ DOURADOS } & 38.467 & AMAMBAI & 7.954 & $21 \%$ \\
\hline & & ITAPORÃ & 6.124 & $16 \%$ \\
\hline & & CAARAPÓ & 5.444 & $14 \%$ \\
\hline & & PARANHOS & 5.247 & $14 \%$ \\
\hline & & TACURU & 3.695 & $10 \%$ \\
\hline & & CORONEL SAPUCAIA & 3.549 & $9 \%$ \\
\hline & & DOURADINA & 1.344 & $3 \%$ \\
\hline & & ANTÔNIO JOÃO & 1.076 & $3 \%$ \\
\hline & & LAGUNA CARAPÃ & 918 & $2 \%$ \\
\hline & & RIO BRILHANTE & 656 & $2 \%$ \\
\hline & & JUTI & 591 & $2 \%$ \\
\hline & & PONTA PORÃ & 565 & $1 \%$ \\
\hline & & ELDORADO & 539 & $1 \%$ \\
\hline & & MARACAJU & 361 & $1 \%$ \\
\hline & & SETE QUEDAS & 284 & $1 \%$ \\
\hline & & NAVIRAÍ & 119 & $0 \%$ \\
\hline \multirow[t]{13}{*}{ TERESINA } & 33.928 & ALTAMIRA & 6.117 & $18 \%$ \\
\hline & & GRAJAÚ & 6.109 & $18 \%$ \\
\hline & & FERNANDO FALCÃO & 5.563 & $16 \%$ \\
\hline & & JENIPAPO DOS VIEIRAS & 5.332 & $16 \%$ \\
\hline & & BARRA DO CORDA & 4.829 & $14 \%$ \\
\hline & & ARAME & 2.246 & $7 \%$ \\
\hline & & MONTES ALTOS & 1.854 & $5 \%$ \\
\hline & & TUNTUM & 799 & $2 \%$ \\
\hline & & BURITICUPU & 389 & $1 \%$ \\
\hline & & LAJEADO NOVO & 224 & $1 \%$ \\
\hline & & URUÇUÍ & 191 & $1 \%$ \\
\hline & & QUEIMADA NOVA & 186 & $1 \%$ \\
\hline & & FORMOSA DA SERRA NEGRA & 90 & $0 \%$ \\
\hline \multirow[t]{13}{*}{ PORTO VELHO } & 30.789 & GUAJARÁ-MIRIM & 8.713 & $28 \%$ \\
\hline & & NOVA MAMORÉ & 8.091 & $26 \%$ \\
\hline & & JI-PARANÁ & 4.475 & $15 \%$ \\
\hline & & HUMAITÁ & 2.174 & $7 \%$ \\
\hline & & SÃO FRANCISCO DO GUAPORÉ & 1.583 & $5 \%$ \\
\hline & & ESPIGÃO D'OESTE & 1.546 & $5 \%$ \\
\hline & & GOVERNADOR JORGE TEIXEIRA & 1.398 & $5 \%$ \\
\hline & & CACOAL & 1.279 & $4 \%$ \\
\hline & & VILHENA & 1.097 & $4 \%$ \\
\hline & & SÃO MIGUEL DO GUAPORÉ & 345 & $1 \%$ \\
\hline & & JARU & 56 & $0 \%$ \\
\hline & & CACAULÂNDIA & 25 & $0 \%$ \\
\hline & & ALVORADA D'OESTE & 8 & $0 \%$ \\
\hline CAMPO GRANDE & 30.173 & AQUIDAUANA & 7.691 & $25 \%$ \\
\hline
\end{tabular}




\begin{tabular}{|c|c|c|c|c|}
\hline & & \multirow[b]{2}{*}{ DOURADOS } & \multirow[b]{2}{*}{7.216} & \multirow[b]{2}{*}{$24 \%$} \\
\hline & & & & \\
\hline & & MIRANDA & 7.034 & $23 \%$ \\
\hline & & SIDROLÂNDIA & 1.916 & $6 \%$ \\
\hline & & DOIS IRMÃOS DO BURITI & 1.884 & $6 \%$ \\
\hline & & PORTO MURTINHO & 1.836 & $6 \%$ \\
\hline & & NIOAQUE & 1.684 & $6 \%$ \\
\hline & & BELA VISTA & 464 & $2 \%$ \\
\hline & & BODOQUENA & 141 & $0 \%$ \\
\hline & & CORUMBÁ & 141 & $0 \%$ \\
\hline & & JARDIM & 86 & $0 \%$ \\
\hline & & JARAGUARI & 81 & $0 \%$ \\
\hline \multirow[t]{20}{*}{ CUIABÁ } & 28.761 & GAÚCHA DO NORTE & 4.925 & $17 \%$ \\
\hline & & COMODORO & 4.209 & $15 \%$ \\
\hline & & JUÍNA & 4.137 & $14 \%$ \\
\hline & & GENERAL CARNEIRO & 4.012 & $14 \%$ \\
\hline & & APIACÁS & 2.484 & $9 \%$ \\
\hline & & SÃO JOSÉ DO XINGU & 2.161 & $8 \%$ \\
\hline & & SANTA TEREZINHA & 2.091 & $7 \%$ \\
\hline & & TANGARÁ DA SERRA & 1.391 & $5 \%$ \\
\hline & & RONDONÓPOLIS & 1.068 & $4 \%$ \\
\hline & & SANTO ANTÔNIO DO LEVERGER & 515 & $2 \%$ \\
\hline & & CONFRESA & 344 & $1 \%$ \\
\hline & & CAMPOS DE JÚLIO & 320 & $1 \%$ \\
\hline & & ÁGUA BOA & 298 & $1 \%$ \\
\hline & & NOBRES & 242 & $1 \%$ \\
\hline & & PONTES E LACERDA & 145 & $1 \%$ \\
\hline & & CANABRAVA DO NORTE & 130 & $0 \%$ \\
\hline & & BARÃO DE MELGAÇO & 125 & $0 \%$ \\
\hline & & NOVO SÃO JOAQUIM & 124 & $0 \%$ \\
\hline & & DIAMANTINO & 20 & $0 \%$ \\
\hline & & PLANALTO DA SERRA & 20 & $0 \%$ \\
\hline \multirow[t]{9}{*}{ RIO BRANCO } & 26.836 & SANTA ROSA DO PURUS & 6.950 & $26 \%$ \\
\hline & & FEIJÓ & 5.525 & $21 \%$ \\
\hline & & PAUINI & 3.903 & $15 \%$ \\
\hline & & BOCA DO ACRE & 3.215 & $12 \%$ \\
\hline & & TARAUACÁ & 2.987 & $11 \%$ \\
\hline & & SENA MADUREIRA & 1.563 & $6 \%$ \\
\hline & & CRUZEIRO DO SUL & 1.207 & $4 \%$ \\
\hline & & ASSIS BRASIL & 842 & $3 \%$ \\
\hline & & MANOEL URBANO & 645 & $2 \%$ \\
\hline \multirow[t]{6}{*}{ SALVADOR } & 15.817 & PORTO SEGURO & 4.425 & $28 \%$ \\
\hline & & ABARÉ & 4.134 & $26 \%$ \\
\hline & & GLÓRIA & 2.843 & $18 \%$ \\
\hline & & BANZAÊE & 2.580 & $16 \%$ \\
\hline & & EUCLIDES DA CUNHA & 764 & $5 \%$ \\
\hline & & RODELAS & 476 & $3 \%$ \\
\hline
\end{tabular}




\begin{tabular}{|c|c|c|c|c|}
\hline & & & & \\
\hline & & IBOTIRAMA & 430 & $3 \%$ \\
\hline & & PAULO AFONSO & 124 & $1 \%$ \\
\hline & & SERRA DO RAMALHO & 41 & $0 \%$ \\
\hline \multirow[t]{7}{*}{ ITABUNA } & \multirow[t]{7}{*}{14.906} & ILHÉUS & 4.909 & $33 \%$ \\
\hline & & SANTA CRUZ CABRÁLIA & 3.578 & $24 \%$ \\
\hline & & ITAJU DO COLÔNIA & 2.283 & $15 \%$ \\
\hline & & PAU BRASIL & 2.135 & $14 \%$ \\
\hline & & BUERAREMA & 1.582 & $11 \%$ \\
\hline & & BELMONTE & 278 & $2 \%$ \\
\hline & & CAMACAN & 141 & $1 \%$ \\
\hline \multirow[t]{2}{*}{ MAMANGUAPE } & \multirow[t]{2}{*}{14.384} & MARCAÇÃO & 8.827 & $61 \%$ \\
\hline & & BAÍA DA TRAIÇÃO & 5.558 & $39 \%$ \\
\hline \multirow[t]{9}{*}{ MACEIÓ } & \multirow[t]{9}{*}{13.972} & JOAQUIM GOMES & 4.760 & $34 \%$ \\
\hline & & PALMEIRA DOS ÍNDIOS & 4.647 & $33 \%$ \\
\hline & & PARICONHA & 1.616 & $12 \%$ \\
\hline & & SÃO SEBASTIÃO & 1.334 & $10 \%$ \\
\hline & & MATRIZ DE CAMARAGIBE & 857 & $6 \%$ \\
\hline & & FEIRA GRANDE & 473 & $3 \%$ \\
\hline & & TRAIPU & 107 & $1 \%$ \\
\hline & & COLÔNIA LEOPOLDINA & 99 & $1 \%$ \\
\hline & & NOVO LINO & 79 & $1 \%$ \\
\hline \multirow[t]{2}{*}{ SORRISO } & \multirow[t]{2}{*}{13.001} & FELIZ NATAL & 12.319 & $95 \%$ \\
\hline & & NOVA UBIRATÃ & 682 & $5 \%$ \\
\hline \multirow[t]{3}{*}{ SERRA TALHADA } & \multirow[t]{3}{*}{12.663} & CARNAUBEIRA DA PENHA & 9.910 & $78 \%$ \\
\hline & & FLORESTA & 2.737 & $22 \%$ \\
\hline & & BETÂNIA & 17 & $0 \%$ \\
\hline \multirow[t]{5}{*}{ SOBRAL } & \multirow[t]{5}{*}{12.476} & ITAREMA & 7.758 & $62 \%$ \\
\hline & & MONSENHOR TABOSA & 2.249 & $18 \%$ \\
\hline & & PORANGA & 2.050 & $16 \%$ \\
\hline & & TAMBORIL & 311 & $2 \%$ \\
\hline & & ACARAÚ & 108 & $1 \%$ \\
\hline \multirow[t]{5}{*}{ IMPERATRIZ } & \multirow[t]{5}{*}{11.524} & AMARANTE DO MARANHÃO & 5.288 & $46 \%$ \\
\hline & & TOCANTINÓPOLIS & 3.004 & $26 \%$ \\
\hline & & BOM JESUS DO TOCANTINS & 2.117 & $18 \%$ \\
\hline & & BOM JESUS DAS SELVAS & 1.003 & $9 \%$ \\
\hline & & RONDON DO PARÁ & 112 & $1 \%$ \\
\hline \multirow[t]{3}{*}{ MONTES CLAROS } & \multirow[t]{3}{*}{11.460} & SÃO JOÃO DAS MISSÕES & 8.969 & $78 \%$ \\
\hline & & ITACARAMBI & 2.381 & $21 \%$ \\
\hline & & MATIAS CARDOSO & 110 & $1 \%$ \\
\hline \multirow[t]{2}{*}{ SANTO ANTÔNIO DO IÇÁ } & \multirow[t]{2}{*}{11.256} & AMATURÁ & 6.095 & $54 \%$ \\
\hline & & TONANTINS & 5.160 & $46 \%$ \\
\hline \multirow[t]{5}{*}{ BELÉM } & \multirow[t]{5}{*}{10.983} & SANTARÉM & 4.845 & $44 \%$ \\
\hline & & PARAGOMINAS & 2.242 & $20 \%$ \\
\hline & & MOJU & 1.305 & $12 \%$ \\
\hline & & SENADOR JOSÉ PORFÍRIO & 1.152 & $10 \%$ \\
\hline & & BRASIL NOVO & 889 & $8 \%$ \\
\hline
\end{tabular}




\begin{tabular}{|c|c|c|c|c|}
\hline & & TOMÉ-AÇU & 436 & $4 \%$ \\
\hline & & PORTO DE MOZ & 74 & $1 \%$ \\
\hline & & PORTEL & 40 & $0 \%$ \\
\hline \multirow[t]{6}{*}{ SINOP } & \multirow[t]{6}{*}{9.767} & JUARA & 6.512 & $67 \%$ \\
\hline & & PEIXOTO DE AZEVEDO & 1.901 & $19 \%$ \\
\hline & & MARCELÂNDIA & 848 & $9 \%$ \\
\hline & & MATUPÁ & 248 & $3 \%$ \\
\hline & & TABAPORÃ & 227 & $2 \%$ \\
\hline & & GUARANTÃ DO NORTE & 32 & $0 \%$ \\
\hline \multirow[t]{4}{*}{ TANGARÁ DA SERRA } & \multirow[t]{4}{*}{9.347} & BRASNORTE & 5.852 & $63 \%$ \\
\hline & & SAPEZAL & 1.435 & $15 \%$ \\
\hline & & BARRA DO BUGRES & 1.127 & $12 \%$ \\
\hline & & CAMPO NOVO DO PARECIS & 934 & $10 \%$ \\
\hline \multirow[t]{17}{*}{ PASSO FUNDO } & \multirow[t]{17}{*}{8.929} & TENENTE PORTELA & 1.744 & $20 \%$ \\
\hline & & RONDA ALTA & 1.046 & $12 \%$ \\
\hline & & NONOAI & 1.006 & $11 \%$ \\
\hline & & IRAÍ & 810 & $9 \%$ \\
\hline & & FAXINALZINHO & 717 & $8 \%$ \\
\hline & & ERECHIM & 484 & $5 \%$ \\
\hline & & CONSTANTINA & 470 & $5 \%$ \\
\hline & & TRÊS PALMEIRAS & 461 & $5 \%$ \\
\hline & & GETÚLIO VARGAS & 443 & $5 \%$ \\
\hline & & IBIRAIARAS & 384 & $4 \%$ \\
\hline & & GRAMADO DOS LOUREIROS & 365 & $4 \%$ \\
\hline & & MATO CASTELHANO & 279 & $3 \%$ \\
\hline & & MULITERNO & 205 & $2 \%$ \\
\hline & & RIO DOS ÍNDIOS & 148 & $2 \%$ \\
\hline & & SALTO DO JACUÍ & 148 & $2 \%$ \\
\hline & & SANANDUVA & 119 & $1 \%$ \\
\hline & & CARAZINHO & 101 & $1 \%$ \\
\hline \multirow[t]{4}{*}{ TEFÉ } & \multirow[t]{4}{*}{8.847} & FONTE BOA & 4.091 & $46 \%$ \\
\hline & & ALVARÃES & 2.127 & $24 \%$ \\
\hline & & MARÃ̃ & 1.735 & $20 \%$ \\
\hline & & JURUÁ & 894 & $10 \%$ \\
\hline \multirow[t]{3}{*}{ MACAPÁ } & \multirow[t]{3}{*}{7.265} & OIAPOQUE & 5.690 & $78 \%$ \\
\hline & & PEDRA BRANCA DO AMAPARI & 1.523 & $21 \%$ \\
\hline & & LARANJAL DO JARI & 51 & $1 \%$ \\
\hline \multirow[t]{4}{*}{ XANXERÊ } & \multirow[t]{4}{*}{6.851} & IPUAÇU & 3.691 & $54 \%$ \\
\hline & & CHAPECÓ & 1.650 & $24 \%$ \\
\hline & & ENTRE RIOS & 946 & $14 \%$ \\
\hline & & ABELARDO LUZ & 564 & $8 \%$ \\
\hline \multirow[t]{4}{*}{ REDENÇÃO } & \multirow[t]{4}{*}{6.404} & OURILÂNDIA DO NORTE & 3.267 & $51 \%$ \\
\hline & & CUMARU DO NORTE & 2.119 & $33 \%$ \\
\hline & & PAU D'ARCO & 803 & $13 \%$ \\
\hline & & BANNACH & 214 & $3 \%$ \\
\hline CRUZEIRO DO SUL & 5.836 & MARECHAL THAUMATURGO & 3.613 & $62 \%$ \\
\hline
\end{tabular}




\begin{tabular}{|c|c|c|c|c|}
\hline & & MÂNCIO LIMA & 1.220 & $21 \%$ \\
\hline & & PORTO WALTER & 1.003 & $17 \%$ \\
\hline \multirow[t]{7}{*}{ SÃO LUÍS } & 5.738 & ZÉ DOCA & 1.860 & $32 \%$ \\
\hline & & BOM JARDIM & 1.416 & $25 \%$ \\
\hline & & CENTRO NOVO DO MARANHÃO & 1.246 & $22 \%$ \\
\hline & & MARANHÃOZINHO & 454 & $8 \%$ \\
\hline & & CENTRO DO GUILHERME & 378 & $7 \%$ \\
\hline & & ARAGUANÃ & 339 & $6 \%$ \\
\hline & & SANTA LUZIA & 46 & $1 \%$ \\
\hline \multirow[t]{4}{*}{ SÃO PAULO } & 5.400 & BOA VISTA & 3.305 & $61 \%$ \\
\hline & & TUCURUÍ & 1.707 & $32 \%$ \\
\hline & & SETE BARRAS & 216 & $4 \%$ \\
\hline & & PARIQUERA-AÇU & 172 & $3 \%$ \\
\hline \multirow[t]{5}{*}{ PALMAS } & 5.315 & FORMOSO DO ARAGUAIA & 1.950 & $37 \%$ \\
\hline & & LAGOA DA CONFUSÃO & 1.812 & $34 \%$ \\
\hline & & PIUM & 1.176 & $22 \%$ \\
\hline & & SANDOLÂNDIA & 292 & $5 \%$ \\
\hline & & PEDRO AFONSO & 86 & $2 \%$ \\
\hline \multirow[t]{3}{*}{ PAULO AFONSO } & 5.153 & JATOBÁ & 4.213 & $82 \%$ \\
\hline & & PETROLÂNDIA & 824 & $16 \%$ \\
\hline & & ÁGUA BRANCA & 116 & $2 \%$ \\
\hline \multirow[t]{3}{*}{ TUCURUÍ } & 5.092 & NOVO REPARTIMENTO & 4.711 & $93 \%$ \\
\hline & & GOIANÉSIA DO PARÁ & 235 & $5 \%$ \\
\hline & & BAIÃO & 146 & $3 \%$ \\
\hline VITÓRIA & 4.950 & ARACRUZ & 4.950 & $100 \%$ \\
\hline \multirow[t]{5}{*}{ ARAGUAÍNA } & 4.923 & GOIATINS & 1.937 & $39 \%$ \\
\hline & & PARAUAPEBAS & 1.166 & $24 \%$ \\
\hline & & SÃO GERALDO DO ARAGUAIA & 943 & $19 \%$ \\
\hline & & MARABÁ & 458 & $9 \%$ \\
\hline & & SANTA FÉ DO ARAGUAIA & 420 & $9 \%$ \\
\hline LARANJEIRAS DO SUL & 4.345 & NOVA LARANJEIRAS & 4.345 & $100 \%$ \\
\hline TARAUACÁ & 4.293 & JORDÃO & 4.293 & $100 \%$ \\
\hline \multirow[t]{2}{*}{ TENENTE PORTELA } & 4.242 & REDENTORA & 4.050 & $95 \%$ \\
\hline & & VICENTE DUTRA & 192 & $5 \%$ \\
\hline \multirow[t]{2}{*}{ RONDONÓPOLIS } & 4.013 & SANTO ANTÔNIO DO LESTE & 2.335 & $58 \%$ \\
\hline & & POXORÉU & 1.678 & $42 \%$ \\
\hline MUNDO NOVO & 3.837 & JAPORÃ & 3.837 & $100 \%$ \\
\hline JI-PARANÁ & 3.783 & RONDOLÂNDIA & 3.783 & $100 \%$ \\
\hline \multirow[t]{8}{*}{ PORTO ALEGRE } & 3.780 & CAMAQUÃ & 2.268 & $60 \%$ \\
\hline & & BARRA DO RIBEIRO & 457 & $12 \%$ \\
\hline & & RIO GRANDE & 297 & $8 \%$ \\
\hline & & SANTA MARIA & 219 & $6 \%$ \\
\hline & & LAJEADO & 137 & $4 \%$ \\
\hline & & MARIANA PIMENTEL & 91 & $2 \%$ \\
\hline & & TORRES & 91 & $2 \%$ \\
\hline & & CAÇAPAVA DO SUL & 73 & $2 \%$ \\
\hline
\end{tabular}




\begin{tabular}{|c|c|c|c|c|}
\hline & & & & \\
\hline & & CAPIVARI DO SUL & 50 & $1 \%$ \\
\hline & & CRISTAL & 32 & $1 \%$ \\
\hline & & MAQUINÉ & 24 & $1 \%$ \\
\hline & & TABAÍ & 23 & $1 \%$ \\
\hline & & PALMARES DO SUL & 17 & $0 \%$ \\
\hline \multirow[t]{2}{*}{ TEIXEIRA DE FREITAS } & \multirow[t]{2}{*}{3.755} & PRADO & 3.345 & $89 \%$ \\
\hline & & ITAMARAJU & 410 & $11 \%$ \\
\hline \multirow[t]{5}{*}{ LONDRINA } & \multirow[t]{5}{*}{3.526} & TAMARANA & 2.066 & $59 \%$ \\
\hline & & SÃO JERÔNIMO DA SERRA & 1.130 & $32 \%$ \\
\hline & & SANTA AMÉLIA & 178 & $5 \%$ \\
\hline & & RIBEIRÃO DO PINHAL & 128 & $4 \%$ \\
\hline & & ABATIÁ & 24 & $1 \%$ \\
\hline \multirow[t]{2}{*}{ PROPRIÁ } & \multirow[t]{2}{*}{3.483} & PORTO REAL DO COLÉGIO & 3.338 & $96 \%$ \\
\hline & & SÃO BRÁS & 145 & $4 \%$ \\
\hline \multirow[t]{3}{*}{ CACOAL } & \multirow[t]{3}{*}{3.382} & ALTA FLORESTA D'OESTE & 2.298 & $68 \%$ \\
\hline & & PIMENTA BUENO & 972 & $29 \%$ \\
\hline & & PARECIS & 112 & $3 \%$ \\
\hline \multirow[t]{3}{*}{ ALTAMIRA } & \multirow[t]{3}{*}{3.191} & ANAPU & 2.184 & $68 \%$ \\
\hline & & URUARÁ & 582 & $18 \%$ \\
\hline & & VITÓRIA DO XINGU & 425 & $13 \%$ \\
\hline \multirow[t]{2}{*}{ RIO DO SUL } & \multirow[t]{2}{*}{3.189} & VITOR MEIRELES & 1.747 & $55 \%$ \\
\hline & & JOSÉ BOITEUX & 1.443 & $45 \%$ \\
\hline \multirow[t]{4}{*}{ CASCAVEL } & \multirow[t]{4}{*}{3.144} & TERRA ROXA & 1.151 & $37 \%$ \\
\hline & & LARANJEIRAS DO SUL & 911 & $29 \%$ \\
\hline & & ESPIGÃO ALTO DO IGUAÇU & 548 & $17 \%$ \\
\hline & & DIAMANTE D'OESTE & 533 & $17 \%$ \\
\hline \multirow[t]{7}{*}{ FORTALEZA } & \multirow[t]{7}{*}{3.111} & ITAPIPOCA & 1.367 & $44 \%$ \\
\hline & & CRATEÚS & 750 & $24 \%$ \\
\hline & & ARATUBA & 389 & $13 \%$ \\
\hline & & QUITERIANÓPOLIS & 311 & $10 \%$ \\
\hline & & NOVO ORIENTE & 145 & $5 \%$ \\
\hline & & BOA VIAGEM & 75 & $2 \%$ \\
\hline & & CANINDÉ & 75 & $2 \%$ \\
\hline \multirow[t]{2}{*}{ CAPITÃO POÇO } & \multirow[t]{2}{*}{2.933} & NOVA ESPERANÇA DO PIRIÁ & 2.899 & $99 \%$ \\
\hline & & GARRAFÃO DO NORTE & 34 & $1 \%$ \\
\hline MARAÃ & 2.745 & JAPURÁ & 2.745 & $100 \%$ \\
\hline SÃO FÉLIX DO ARAGUAIA & 2.737 & ALTO BOA VISTA & 2.737 & $100 \%$ \\
\hline \multirow[t]{5}{*}{ PATO BRANCO } & \multirow[t]{5}{*}{2.444} & CHOPINZINHO & 822 & $34 \%$ \\
\hline & & PALMAS & 694 & $28 \%$ \\
\hline & & MANGUEIRINHA & 600 & $25 \%$ \\
\hline & & CORONEL VIVIDA & 214 & $9 \%$ \\
\hline & & CLEVELÂNDIA & 114 & $5 \%$ \\
\hline PRIMAVERA DO LESTE & 2.389 & PARANATINGA & 2.389 & $100 \%$ \\
\hline \multirow[t]{3}{*}{ RODEIO BONITO } & 2.165 & PLANALTO & 1.535 & $71 \%$ \\
\hline & & LIBERATO SALZANO & 423 & $20 \%$ \\
\hline & & ERVAL SECO & 207 & $10 \%$ \\
\hline
\end{tabular}




\begin{tabular}{|c|c|c|c|c|}
\hline \multirow[t]{3}{*}{ BELO HORIZONTE } & \multirow[t]{3}{*}{1.883} & GOVERNADOR VALADARES & 1.509 & $80 \%$ \\
\hline & & BARBACENA & 343 & $18 \%$ \\
\hline & & GUANHÃES & 31 & $2 \%$ \\
\hline \multirow[t]{2}{*}{ TAPEJARA } & \multirow[t]{2}{*}{1.855} & CHARRUA & 1.652 & $89 \%$ \\
\hline & & ÁGUA SANTA & 203 & $11 \%$ \\
\hline \multirow[t]{3}{*}{ JUÍNA } & \multirow[t]{3}{*}{1.734} & ARIPUANÃ & 1.623 & $94 \%$ \\
\hline & & COTRIGUAÇU & 98 & $6 \%$ \\
\hline & & COLNIZA & 13 & $1 \%$ \\
\hline \multirow[t]{2}{*}{ PETROLINA } & \multirow[t]{2}{*}{1.707} & CURAÇÁ & 1.603 & $94 \%$ \\
\hline & & OROCÓ & 104 & $6 \%$ \\
\hline ERECHIM & 1.692 & BENJAMIN CONSTANT DO SUL & 1.692 & $100 \%$ \\
\hline ILHÉUS & 1.682 & UNA & 1.682 & $100 \%$ \\
\hline SANTA INÊS & 1.648 & SÃO JOÃO DO CARÚ & 1.648 & $100 \%$ \\
\hline \multirow{2}{*}{ IVAIPORÃ } & \multirow[t]{2}{*}{1.628} & MANOEL RIBAS & 1.089 & $67 \%$ \\
\hline & & CÂNDIDO DE ABREU & 539 & $33 \%$ \\
\hline PARAGOMINAS & 1.493 & IPIXUNA DO PARÁ & 1.493 & $100 \%$ \\
\hline \multirow[t]{3}{*}{ UMUARAMA } & \multirow{3}{*}{1.430} & GUAÍRA & 1.325 & $93 \%$ \\
\hline & & IVATÉ & 78 & $5 \%$ \\
\hline & & ALTÔNIA & 27 & $2 \%$ \\
\hline \multirow[t]{2}{*}{ VILHENA } & \multirow[t]{2}{*}{1.302} & CHUPINGUAIA & 960 & $74 \%$ \\
\hline & & CORUMBIARA & 341 & $26 \%$ \\
\hline PEDRO AFONSO & 1.242 & ITACAJÁ & 1.242 & $100 \%$ \\
\hline NATAL & 1.210 & JOÃO CÂMARA & 1.210 & $100 \%$ \\
\hline CAPANEMA & 1.161 & SANTA LUZIA DO PARÁ & 1.161 & $100 \%$ \\
\hline EIRUNEPÉ & 1.127 & ENVIRA & 1.127 & $100 \%$ \\
\hline \multirow[t]{5}{*}{ CHAPECÓ } & \multirow[t]{5}{*}{1.089} & SEARA & 586 & $54 \%$ \\
\hline & & SAUDADES & 215 & $20 \%$ \\
\hline & & ARVOREDO & 144 & $13 \%$ \\
\hline & & CUNHA PORÃ & 123 & $11 \%$ \\
\hline & & PAIAL & 22 & $2 \%$ \\
\hline \multirow[t]{2}{*}{ RORAINÓPOLIS } & \multirow[t]{2}{*}{1.049} & CAROEBE & 551 & $53 \%$ \\
\hline & & SÃO JOÃO DA BALIZA & 498 & $47 \%$ \\
\hline SANTO AUGUSTO & 1.044 & SÃO VALÉRIO DO SUL & 1.044 & $100 \%$ \\
\hline TUNTUM & 1.008 & ITAIPAVA DO GRAJAÚ & 1.008 & $100 \%$ \\
\hline URUARÁ & 980 & PLACAS & 980 & $100 \%$ \\
\hline PONTA PORÃ & 970 & ARAL MOREIRA & 970 & $100 \%$ \\
\hline PARINTINS & 953 & NHAMUNDÁ & 953 & $100 \%$ \\
\hline \multirow[t]{3}{*}{ PONTES E LACERDA } & \multirow[t]{3}{*}{923} & CONQUISTA D'OESTE & 492 & $53 \%$ \\
\hline & & VILA BELA DA SANTÍSSIMA TRINDADE & 251 & $27 \%$ \\
\hline & & NOVA LACERDA & 180 & $20 \%$ \\
\hline SÃO JOSÉ DO OURO & 919 & CACIQUE DOBLE & 919 & $100 \%$ \\
\hline \multirow[t]{3}{*}{ TEÓFILO OTONI } & \multirow[t]{3}{*}{873} & BERTÓPOLIS & 503 & $58 \%$ \\
\hline & & SANTA HELENA DE MINAS & 228 & $26 \%$ \\
\hline & & LADAINHA & 142 & $16 \%$ \\
\hline BAURU & 808 & AVAÍ & 808 & $100 \%$ \\
\hline POÇOS DE CALDAS & 779 & SANTA RITA DE CALDAS & 625 & $80 \%$ \\
\hline
\end{tabular}




\begin{tabular}{|c|c|c|c|c|}
\hline & & & & \\
\hline & & CALDAS & 154 & $20 \%$ \\
\hline XINGUARA & 698 & ÁGUA AZUL DO NORTE & 698 & $100 \%$ \\
\hline FOZ DO IGUAÇU & 630 & SÃO MIGUEL DO IGUAÇU & 630 & $100 \%$ \\
\hline TELÊMACO BORBA & 597 & ORTIGUEIRA & 597 & $100 \%$ \\
\hline \multirow[t]{4}{*}{ PARIQUERA-AÇU } & 594 & IGUAPE & 268 & $45 \%$ \\
\hline & & CANANÉIA & 202 & $34 \%$ \\
\hline & & ITARIRI & 110 & $19 \%$ \\
\hline & & ELDORADO & 13 & $2 \%$ \\
\hline \multirow[t]{2}{*}{ SALGUEIRO } & 584 & MIRANDIBA & 516 & $88 \%$ \\
\hline & & BELÉM DO SÃO FRANCISCO & 68 & $12 \%$ \\
\hline GUARAPUAVA & 546 & TURVO & 546 & $100 \%$ \\
\hline \multirow[t]{2}{*}{ AUGUSTINÓPOLIS } & 544 & MAURILÂNDIA DO TOCANTINS & 476 & $87 \%$ \\
\hline & & SÃO BENTO DO TOCANTINS & 68 & $13 \%$ \\
\hline CARAGUATATUBA & 527 & PARATY & 527 & $100 \%$ \\
\hline RONDA ALTA & 522 & ENGENHO VELHO & 522 & $100 \%$ \\
\hline \multirow[t]{2}{*}{ MARABÁ } & 461 & ITUPIRANGA & 242 & $52 \%$ \\
\hline & & BREJO GRANDE DO ARAGUAIA & 219 & $48 \%$ \\
\hline \multirow[t]{2}{*}{ SANTOS } & 461 & PERUÍBE & 408 & $88 \%$ \\
\hline & & BERTIOGA & 54 & $12 \%$ \\
\hline VOLTA REDONDA & 460 & ANGRA DOS REIS & 460 & $100 \%$ \\
\hline ARACAJU & 335 & PORTO DA FOLHA & 335 & $100 \%$ \\
\hline SOROCABA & 334 & TAPIRAÍ & 334 & $100 \%$ \\
\hline NAVIRAÍ & 328 & IGUATEMI & 328 & $100 \%$ \\
\hline GETÚLIO VARGAS & 306 & EREBANGO & 306 & $100 \%$ \\
\hline \multirow[t]{2}{*}{ DIVINÓPOLIS } & 304 & MARTINHO CAMPOS & 251 & $83 \%$ \\
\hline & & ITAPECERICA & 53 & $17 \%$ \\
\hline ROLIM DE MOURA & 300 & ALTO ALEGRE DOS PARECIS & 300 & $100 \%$ \\
\hline GUANHÃES & 289 & CARMÉSIA & 289 & $100 \%$ \\
\hline BRASÍLIA & 267 & MUQUÉM DO SÃO FRANCISCO & 267 & $100 \%$ \\
\hline \multirow[t]{2}{*}{ GOVERNADOR VALADARES } & 266 & RESPLENDOR & 262 & $98 \%$ \\
\hline & & TEÓFILO OTONI & 4 & $2 \%$ \\
\hline REGISTRO & 246 & MIRACATU & 246 & $100 \%$ \\
\hline OURO PRETO DO OESTE & 228 & MIRANTE DA SERRA & 228 & $100 \%$ \\
\hline TIMBÓ & 214 & DOUTOR PEDRINHO & 214 & $100 \%$ \\
\hline ZÉ DOCA & 194 & NOVA OLINDA DO MARANHÃO & 194 & $100 \%$ \\
\hline MEDIANEIRA & 183 & ITAIPULÂNDIA & 183 & $100 \%$ \\
\hline \multirow[t]{3}{*}{ JOINVILLE } & 178 & SÃO FRANCISCO DO SUL & 69 & $38 \%$ \\
\hline & & BALNEÁRIO BARRA DO SUL & 64 & $36 \%$ \\
\hline & & GARUVA & 46 & $26 \%$ \\
\hline ITAPORANGA & 167 & BARÃO DE ANTONINA & 167 & $100 \%$ \\
\hline TOLEDO & 160 & SANTA HELENA & 160 & $100 \%$ \\
\hline PARANAGUÁ & 151 & GUARAQUEÇABA & 151 & $100 \%$ \\
\hline CURITIBA & 138 & PARANAGUÁ & 138 & $100 \%$ \\
\hline SANTO ÂNGELO & 137 & SÃO MIGUEL DAS MISSÕES & 137 & $100 \%$ \\
\hline TUBARÃO & 134 & IMARUÍ & 134 & $100 \%$ \\
\hline BARRETOS & 132 & MONTE NEGRO & 132 & $100 \%$ \\
\hline
\end{tabular}




\begin{tabular}{|c|c|c|c|c|}
\hline CERES & 129 & RUBIATABA & 129 & $100 \%$ \\
\hline FLORIANÓPOLIS & 128 & TIJUCAS & 128 & $100 \%$ \\
\hline MAFRA & 127 & ITAIÓPOLIS & 127 & $100 \%$ \\
\hline IPATINGA & 119 & AÇUCENA & 119 & $100 \%$ \\
\hline JACAREZINHO & 118 & TOMAZINA & 118 & $100 \%$ \\
\hline SANTANA DO IPANEMA & 116 & INHAPI & 116 & $100 \%$ \\
\hline IRATI & 112 & INÁCIO MARTINS & 112 & $100 \%$ \\
\hline SÃO JOÃO BATISTA & 110 & MAJOR GERCINO & 110 & $100 \%$ \\
\hline ARAPIRACA & 95 & CAMPO GRANDE & 95 & $100 \%$ \\
\hline MÃE DO RIO & 92 & AURORA DO PARÁ & 92 & $100 \%$ \\
\hline SETE LAGOAS & 75 & POMPÉU & 75 & $100 \%$ \\
\hline CASTANHAL & 74 & SANTA MARIA DO PARÁ & 74 & $100 \%$ \\
\hline VALENÇA & 72 & CAMAMU & 72 & $100 \%$ \\
\hline TRÊS LAGOAS & 71 & BRASILÂNDIA & 71 & $100 \%$ \\
\hline TRAMANDAÍ & 64 & CARAÁ & 64 & $100 \%$ \\
\hline JAÚ & 62 & ITAPORANGA & 62 & $100 \%$ \\
\hline TAQUARA & 55 & RIOZINHO & 55 & $100 \%$ \\
\hline ITAMBACURI & 40 & CAMPANÁRIO & 40 & $100 \%$ \\
\hline IJUÍ & 37 & SANTO ÂNGELO & 37 & $100 \%$ \\
\hline
\end{tabular}

